PALABRAS CLAVE

Crecimiento económico

Producto interno bruto

Balanza de pagos

Exportaciones

Importaciones

Cambio tecnológico

Producción

Análisis económico

Brasil

João Prates Romero

Alumno de la maestría en Economía

del Centro de Desarrollo y Planificación

Regional/Universidad Federal de Minas

Gerais, becado por la Coordinadora de

Perfeccionamiento de Personal de nivel

superior (Brasil).

œ joaopr@cedeplar.ufmg.br

Fabrício Silveira

Alumno de la maestría en Economía

del Centro de Desarrollo y Planificación

Regional/Universidad Federal de Minas

Gerais, becado por el Consejo Nacional

de Desarrollo Científico y Tecnológico

(CNPq) (Brasil).

$\nsim$ silveira@cedeplar.ufmg.br

Frederico G. Jayme Jr.

Profesor del Departamento de Economía y del Centro de Desarrollo y Planificación Regional/Universidad Federal de Minas

Gerais.

ononzaga@cedeplar.ufmg.br
REVISTA CEPAL 105 DICIEMBRE 2011

\section{Brasil: Cambio estructural y crecimiento con restricción de balanza de pagos}

\author{
João Prates Romero, Fabrício Silveira y Frederico G. Jayme Jr.
}

0

e sostiene que las diferencias en las tasas de crecimiento del producto se vinculan a las diferencias en las elasticidades-renta que, a su vez, son determinadas por el grado de intensidad tecnológica de la producción nacional. Para corroborar esta hipótesis se efectuaron pruebas estadísticas. Se estimaron las elasticidades hipotéticas a fin de demostrar su validez en el caso brasileño: i) básica, ii) ampliada con flujos de capitales, y iii) implícita. Se emplearon técnicas de cointegración y un vector de corrección de errores en las estimaciones de las elasticidades reales para cada categoría tecnológica de producto de la pauta comercial brasileña. Los resultados se corroboraron mediante la investigación de funciones de impulso-respuesta y la descomposición de los errores finales de predicción, confirmándose que los bienes de mayor intensidad tecnológica presentan una elasticidadrenta más elevada. Así, según la ley de Thirlwall, una mayor producción nacional de esos bienes impulsaría el crecimiento. 


\section{I}

\section{Introducción}

Existe una vasta literatura en que se señala el papel preponderante de las elasticidades del comercio exterior en la determinación del producto brasileño (Jayme Jr., 2003; Santos, Lima y Carvalho, 2005; Porcile y Lima, 2006; Vieira y Holland, 2006; Carvalho y Lima, 2008). A pesar de que en estos estudios se haya convergido en la aceptación de la restricción externa al crecimiento del país, como se propone en la ley de Thirlwall (Thirlwall, 1979), hasta ahora se ha avanzado poco en la comprensión de los mecanismos que determinan las elasticidades. Sin embargo, en algunos estudios recientes se ha demostrado que la estructura productiva ejerce una importante influencia en estas elasticidades (Gouvêa y Lima, 2009; Araujo y Lima, 2007) ${ }^{1}$. En el presente artículo se plantea que las diferencias en las tasas de crecimiento del producto se relacionan con las diferencias en las elasticidadesrenta, las que a su vez son determinadas por el grado de intensidad tecnológica de la producción nacional. Se sostiene la tesis de que el cambio estructural, entendido como la variación de la contribución de los sectores al producto interno bruto (PIB), contribuye también a la relajación de la restricción externa al crecimiento al reflejarse en el patrón de especialización del comercio exterior. En este argumento se encuentra implícita la hipótesis de que los diferentes sectores producen bienes con distintas elasticidades, lo que convalida un modelo multisectorial de la ley de Thirlwall, de modo que la

\footnotetext{
${ }^{1}$ Este resultado fue posible al considerar un modelo multisectorial de la ley de Thirlwall, donde cada uno de los diversos sectores posee una elasticidad-renta de su determinada producción. Por lo tanto, la elasticidad-renta total de la economía es el resultado de la suma de las elasticidades de esos distintos sectores, ponderada por su participación en el producto nacional. De esta manera, la modificación en la composición de la estructura productiva afecta también a la elasticidad total de la economía.
}

modificación en su participación en el producto se refleja en la elasticidad nacional ${ }^{2}$.

Para corroborar tales hipótesis se realizaron diversas pruebas estadísticas. En primer lugar, a partir de bases de datos distintas se estimaron las elasticidades hipotéticas: i) básica (McCombie, 1997); ii) ampliada con flujos de capitales (Moreno-Brid, 2003), y iii) implícita (Atesoglu, 1997). Se utilizaron técnicas de cointegración y un vector de corrección de errores (VEC) en las estimaciones de las elasticidades reales para cada categoría tecnológica de producto de la pauta comercial brasileña. Dichas categorías se construyeron sobre la base de la clasificación de los productos por nivel tecnológico, de acuerdo con Lall (2001). Los resultados de los análisis son corroborados por la investigación de innovaciones en el modelo, mediante funciones de impulso-respuesta y la descomposición de los errores finales de predicción. Finalmente, se procuró establecer la evolución de las elasticidades del comercio brasileño en concordancia con la metodología propuesta por Gouvêa y Lima (2009).

Además de esta Introducción, el artículo se divide en cuatro secciones adicionales. En la sección II se discuten los elementos de matriz kaldoriana-keynesiana de crecimiento bajo restricción externa. En la sección III se presenta un breve relato sobre la reciente especialización de la estructura productiva brasileña en productos con bajo contenido tecnológico, lo que se puede observar a través de la especialización de su pauta comercial. En la sección IV se presenta la metodología de prueba de los datos brasileños y los resultados de las estimaciones, mientras que las conclusiones se entregan en la sección V.

\footnotetext{
${ }^{2}$ Teóricamente, el tipo de estructura productiva de cada país determina su pauta de comercio internacional, lo que favorece la exportación de bienes de mayor contenido tecnológico (de alta elasticidad-renta) y la importación de bienes más básicos (de baja elasticidad-renta).
} 


\section{II}

\section{Marco teórico}

Entender las causas del crecimiento económico desigual fue siempre uno de los grandes temas de estudio de la matriz teórica kaldoriana-keynesiana (Kaldor, 1966; Thirlwall, 1979; McCombie y Thirlwall, 1994). Tales estudios tienen en común el énfasis en la demanda como motor del sistema económico, mientras que las diferencias de crecimiento entre los diversos países serían el fruto de distintos ritmos de crecimiento de la demanda, que cambiarían de un país a otro de acuerdo con las restricciones impuestas a esta. En este sentido, Thirlwall (1979) enfatiza el papel de la restricción de la balanza de pagos en el desempeño económico, debido a la exigencia de un equilibrio externo a largo plazo. De hecho, ante la imposibilidad del financiamiento continuo de los desequilibrios en la balanza de pagos, surge la necesidad de ajuste de la demanda agregada. De este modo se desincentiva la inversión y se reduce el progreso tecnológico, de manera que los bienes propios del país, comparados con los bienes extranjeros, se tornan menos atractivos. Ello empeora la balanza de pagos y así sucesivamente, dando inicio a un círculo vicioso. Por el contrario, si un país se muestra capaz de expandir la demanda hasta alcanzar la capacidad productiva existente, sin la irrupción de dificultades en la balanza de pagos, es posible que la presión de la demanda sobre la capacidad haga crecer la tasa de expansión de esta última al incentivar la inversión, el progreso tecnológico y la productividad (McCombie y Thirlwall, 1994).

Por lo tanto, el incentivo a la demanda sería responsable del desencadenamiento de un círculo virtuoso de crecimiento, que tendería a aumentar la productividad global de la economía debido a la migración de los factores hacia sectores de mayor productividad (manufacturas) y a la intensificación del concepto del aprendizaje práctico (learn-by-doing) (Kaldor, 1966). El incremento de la demanda altera la lógica sectorial de incentivos en la economía, lo que favorece a algunos sectores en detrimento de otros. Los sectores beneficiados son, sobre todo, aquellos de mayor elasticidad-renta de la demanda (mayor contenido tecnológico, de acuerdo con la hipótesis por confirmar). Además, ellos son generalmente los sectores económicos de retornos crecientes, donde el aumento de su participación en el producto, con la consiguiente movilidad de los factores hacia su producción, hace que la economía se vuelva más productiva. La inversión es la variable central en la propulsión del crecimiento. A su vez, la importancia de la balanza de pagos se da por la magnitud de su incentivo o falta de este al crecimiento de la inversión.

Ese argumento condujo a la formulación de las teorías del crecimiento liderado por las exportaciones, según las cuales solo a través de las exportaciones sería posible elevar la tasa de crecimiento sin un deterioro de la balanza de pagos.

\section{El modelo de restricción externa al crecimiento económico}

Teniendo en cuenta la destacada importancia del equilibrio externo para el crecimiento de la demanda y del producto, Thirlwall —en su trabajo precursor de 1979desarrolló un modelo de crecimiento bajo restricción externa en el que este se relaciona intrínsecamente con las elasticidades-renta de las exportaciones e importaciones.

En su modelo, el equilibrio de la balanza de pagos en moneda local es consecuencia de:

$$
P_{d} X=P_{f} M E
$$

donde $E$ es el tipo de cambio. Las importaciones $(M)$ son función de la relación de precios ponderada por la elasticidad-precio de la demanda por importaciones $(\psi<0)$ y por la elasticidad-renta de la demanda por importaciones $(\pi>0)$, tal como se aprecia en la siguiente ecuación ${ }^{3}$ :

$$
M=a\left(\frac{P_{f} E}{P_{d}}\right)^{\psi} Y^{\pi}
$$

De manera similar, las exportaciones son función del tipo de cambio real y de la renta externa, de modo que la elasticidad-renta de las exportaciones se da por $\varepsilon>0$, y la elasticidad-precio de la demanda de exportaciones por $\eta<0$, ambas en moneda extranjera:

\footnotetext{
${ }^{3}$ Se asume que la elasticidad-precio de la demanda por importaciones y exportaciones es igual a su elasticidad-precio cruzada, es decir, $\psi=\phi$ y $\eta=\tau$ respectivamente.
} 


$$
X=b\left(\frac{P_{d}}{P_{f} E}\right)^{\eta} Z^{\varepsilon}
$$

$\mathrm{Al}$ realizar una transformación lineal de las ecuaciones, sometiéndolas a la condición inicial de equilibrio en la balanza de pagos, se obtiene la tasa de crecimiento de la renta interna consistente con el equilibrio de la balanza de pagos (McCombie y Thirlwall, 1994, págs. 234 y 235):

$$
y_{B}=\frac{(1+\eta+\psi)\left(p_{d}-p_{f}-e\right)+\varepsilon z}{\pi}
$$

La ecuación (4) posee varias implicancias: i) la inflación interna superior a la externa reduce la tasa de crecimiento con equilibrio de la balanza de pagos si $|\psi+\eta|>1$; ii) la depreciación cambiaria $(e>0)$ tiende a aumentar la tasa de crecimiento con equilibrio de la balanza de pagos si $|\psi+\eta|>1$; (teorema o condición de Marshall-Lerner); iii) la mayor tasa de crecimiento de la renta mundial aumenta la tasa de crecimiento con equilibrio de la balanza de pagos, y iv) cuanto mayor sea la elasticidad-renta de la demanda por importaciones $(\pi)$, menor será la tasa de crecimiento con equilibrio de la balanza de pagos.

Sin embargo, al aceptar la validez de la paridad del poder adquisitivo (PPA) a largo plazo, es decir, al asumir que no hay modificación de precios relativos, con la inflación interna igual a la internacional $\left(p_{d t}-p_{f t}-e_{t}=0\right)$, la ecuación puede reducirse a la relación inicialmente propuesta por Thirlwall (1979), que equivale a la regla de crecimiento propuesta por Harrod (1933):

$$
y_{B}=\frac{\varepsilon z}{\pi}=\frac{x}{\pi}
$$

Las evidencias empíricas proporcionadas por McCombie y Thirlwall (1994) confirman esa relación, demostrando que para aumentar su ritmo de crecimiento un país debe superar previamente la restricción de la balanza de pagos. La superación de dicha restricción, con la consiguiente viabilización de una mayor tasa de crecimiento, ocurre a su vez como consecuencia de políticas de estímulo al incremento de las elasticidadesrenta de las exportaciones, en concomitancia con la reducción de las elasticidades-renta de las importaciones. Sin embargo, para aproximar ese referente a la realidad de los países en desarrollo se deben considerar nuevos componentes explicativos, en especial los flujos de capitales, las alteraciones en el tipo de cambio y los pagos de servicios financieros de la deuda (Thirlwall y Hussain, 1982; McCombie y Thirlwall, 1997).

En primer lugar, en los países en desarrollo las corrientes de capital son de gran importancia, ya que permiten el mantenimiento temporario de déficits en la cuenta corriente. De esa manera, los países que presentan déficits comerciales podrían mantener su crecimiento siempre que lograran financiar este déficit con la captación de capitales. No obstante, cabe destacar que la entrada de capitales genera también un pasivo que puede deprimir el ritmo de crecimiento del producto, debido a que estos flujos necesitan amortizarse. Junto con esta hipótesis es importante agregar al modelo el envío de intereses al exterior, puesto que —en algún momento — resulta imperativo tener un superávit en la balanza comercial para el pago del servicio del endeudamiento externo. Es decir, la acumulación de deuda externa también puede generar la necesidad de contracción de la demanda (renta) interna para que se alcance un superávit en la balanza de pagos a objeto de pagar la deuda, lo que reduce el ritmo de crecimiento (Moreno-Brid, 2003; Barbosa-Filho, 2001).

Mediante la incorporación de esos componentes, Moreno-Brid (2003) llega a la siguiente ecuación de equilibrio de la balanza de pagos:

$$
P_{d} X_{t}+P_{d} F+P_{d} R=P_{f} M_{t} E_{t}
$$

donde $F$ representa el flujo de capitales y $R$ el valor real de los servicios de capital. Se incluye también una ponderación $\theta 1$ para la participación de las exportaciones y $\theta 2=(1-\theta 1)$ para la participación de los capitales en los ingresos. En tasas de crecimiento se obtiene:

$$
\begin{gathered}
m_{t}+p_{f t}+e_{t}=\theta 1\left(p_{d t}+x\right)-\theta 2\left(p_{d t}+r\right)+ \\
(1-\theta 1-\theta 2)\left(p_{d t}+f\right)
\end{gathered}
$$

donde $r$ es la variación del pago de intereses netos, $f$ la variación de flujos de capitales y $\theta 1$ y $\theta 2$ son las siguientes razones medidas en el período inicial:

$$
\begin{gathered}
\theta 1=\frac{P_{d} X}{P_{f} E M} \\
\theta 2=\frac{P_{d} R}{P_{f} E M}
\end{gathered}
$$


Finalmente, se introduce también la restricción de endeudamiento sustentable, $F / Y=k$, que en las tasas de crecimiento se da por:

$$
f+p_{d}=y+p_{d}
$$

Al reemplazar esa restricción en (7) y a partir de las mismas funciones para exportaciones e importaciones, se obtiene la tasa de crecimiento con equilibrio de la balanza de pagos en la presencia de flujos de capitales:

$$
y_{B}^{*}=\frac{(\theta 1 \eta+\psi+1)\left(p_{d}-p_{f}-e\right)+\theta 1 \varepsilon z+\theta 2 r}{\pi-(1-\theta 1+\theta 2)}
$$

El primer término representa el efecto de los cambios de los términos de intercambio; el segundo, el efecto de la demanda de las exportaciones; el tercero, el efecto de los envíos de intereses; y el cuarto, al sustraer en el denominador, el efecto de los flujos de capitales. En la ausencia de flujos de capitales, $\theta 1=1$, de manera que volvemos al resultado inicial de la regla de crecimiento de Harrod (1933).

\section{La estructura productiva y su repercusión en las elasticidades}

La importancia de las elasticidades para el crecimiento incita, por lo tanto, a una investigación más profunda de sus determinantes ${ }^{4}$. Aunque el producto potencial de una economía sea determinado por el nivel de crecimiento de la demanda, el abordaje del crecimiento restringido por la balanza de pagos reitera la relevancia de las características de oferta de los bienes (competitividad no basada en precios). De este modo, si se considera un país que produce una serie de bienes con elasticidades distintas — en que la elasticidad-renta total de la economía se obtiene por medio de la sumatoria de las elasticidades sectoriales, ponderadas por su participación en la estructura productiva-, un cambio de estructura productiva de la economía afecta a la elasticidad-renta de las importaciones y exportaciones, pues los diferentes ritmos de expansión de la demanda en cada sector

\footnotetext{
${ }^{4}$ Implícitamente, en el modelo presentado en la sección anterior se considera un país que produce un único bien con elasticidades dadas e inmutables.
}

conducen a distintas tasas de crecimiento de la economía como un todo.

A partir de esa lógica, Araujo y Lima (2007) desarrollan un modelo que engloba a diferentes sectores, y llegan a lo que los autores denominan Ley de Thirlwall Multisectorial (LTMS). La principal implicancia de este modelo es que los cambios en la participación sectorial de la economía, es decir, en la estructura de producción, repercuten en su tasa de crecimiento, de modo que en un país puede aumentar dicha tasa incluso cuando tal incremento en la renta mundial no se produzca, siempre que logre cambiar la composición sectorial de exportaciones e importaciones (Gouvêa y Lima, 2009).

De acuerdo con el abordaje tradicional de Thirlwall, en la ecuación final del modelo de Araujo y Lima (2007) se observa que la tasa de crecimiento de cada país es directamente proporcional a la tasa de crecimiento de las exportaciones. Sin embargo, esa proporcionalidad posee una relación inversa con la elasticidad-renta sectorial de la demanda de importaciones y una relación directa con la elasticidad-renta sectorial de la demanda de exportaciones. En resumen, la tasa de crecimiento depende de la composición sectorial de la economía.

En búsqueda de validez empírica para esta formulación sectorial de la ley de Thirlwall, los referidos autores estiman las elasticidades de la LTMS para varios países de América Latina y Asia, y verifican que los sectores más intensivos en tecnología presentan una mayor elasticidadrenta, con diferencias menores para las importaciones que para las exportaciones. Concluyen que tanto la ley de Thirlwall original como la multisectorial representan adecuadamente la tasa de crecimiento de la economía. Por último, utilizando las elasticidades-renta sectoriales estimadas como ponderadores relativos, los autores toman las participaciones de cada sector en el comercio exterior para calcular como una media ponderada los cambios anuales de las elasticidades, indicando así el proceso de cambio estructural.

Tales evidencias demuestran que, al profundizar la industrialización y, sobre todo, al elevarse la participación en la economía de los sectores de mayor intensidad tecnológica, se alteran también las elasticidades de las exportaciones e importaciones con una incidencia directa en las tasas de crecimiento del producto. Bajo ese marco, en el presente artículo se procura identificar la relación entre las elasticidades y el contenido tecnológico de los bienes que forman parte de la balanza comercial brasileña, para luego analizar los efectos de los cambios estructurales en la tasa de crecimiento del país. 


\section{III}

\section{Restricción externa y estructura productiva en el Brasil: 1962-2010}

En un trabajo empírico realizado para un conjunto de países, McCombie y Thirlwall (1994) concluyeron que existe realmente un deterioro de los términos de intercambio en perjuicio de los países en desarrollo (aunque el efecto real de ese deterioro sea muy pequeño), mientras que los flujos de capitales tienden marginalmente a relajar la restricción, a pesar de que su efecto sea temporario. Tales resultados son ampliamente consistentes con el abordaje estructuralista de Prebisch (2000a y 2000b), del que se derivan las siguientes justificaciones para el fenómeno: i) menor elasticidad-renta de la demanda de los bienes producidos en los países en desarrollo, y ii) mayor elasticidad-renta de la demanda de los bienes producidos en los países centrales.

Carvalho y Lima (2008), al analizar el caso brasileño, verificaron que la expansión observada entre 1930 y 2004 fue compatible con el equilibrio de la balanza de pagos ${ }^{5}$. Asimismo, al estimar la participación de cada uno de los componentes discutidos para la determinación de la tasa de crecimiento, concluyeron que el cambio real es estadísticamente insignificante para el incremento observado, mientras que los flujos de capitales tampoco incidieron en el aumento a largo plazo. La razón de las elasticidades (ley de Thirlwall) fue responsable de la mayor parte del crecimiento, seguida por los términos de intercambio. En el mismo estudio, una prueba de quiebra estructural permitió apreciar - en una submuestra para el período comprendido entre 1930 y 1993 - que la razón de las elasticidades cae del $7 \%$ a apenas un 1,3\% entre 1994 y 2004, lo que demuestra que la pérdida de dinamismo de la economía brasileña en ese lapso se derivó del mantenimiento de una estructura productiva excesivamente apoyada en productos de baja elasticidad-renta de la demanda (o de baja tecnología, según se pretende mostrar), en un período de reorganización de los modelos mundiales del comercio (Jayme Jr. y Resende, 2009). Con respecto a los términos de intercambio, lo que se observa es la tendencia al deterioro entre 1930 y 1993, debido

\footnotetext{
${ }^{5}$ Véanse también Holland, Vieira y Canuto (2004); Ferreira (2001); Bértola, Higachi y Porcile (2002); López y Cruz (2000); Santos, Lima y Carvalho (2005).
}

a su contribución negativa al incremento del producto $(-0,7 \%)$. Ese cuadro se invierte a partir de 1993 , cuando estos términos de intercambio comienzan a dar cuenta de una expansión promedio del producto de $1,7 \%$, probablemente a causa del mayor dinamismo de la economía mundial y la consiguiente mejora de los precios de los productos básicos (commodities).

$\mathrm{Al}$ analizar datos brasileños y de los países de la Organización de Cooperación y Desarrollo Económicos (OCDE), Jayme Jr. y Resende (2009) observan que el Brasil aún no superó la restricción externa al crecimiento, puesto que la balanza comercial de los productos de media y alta intensidad tecnológica concentra grandes déficits desde el inicio de la década de 1990; esto refleja el bajo desarrollo del Sistema Nacional de Innovación (SNI) brasileño y la baja competitividad nacional. Además, luego de la apertura comercial del decenio de 1990, lo que se verificó fue el aumento de la participación de los productos tecnológicos en la pauta de importaciones, sin que haya habido un engrosamiento de las exportaciones de esos productos, lo cual caracteriza una profundización del modelo de inserción internacional periférica. Ello denota que el sector externo brasileño se muestra aún muy vulnerable a las oscilaciones de la demanda internacional, pues sus exportaciones se basan en bienes de bajo tenor tecnológico, es decir, de baja elasticidadrenta. Estos resultados son similares a los obtenidos por Carvalho y Lima (2008).

En los gráficos 1 y 2 se ilustra cómo la estructura productiva brasileña se fue modificando paulatinamente desde 1962. Mientras la participación de las exportaciones de productos primarios se redujo a lo largo del tiempo, la participación de las exportaciones de productos de baja tecnología ganó espacio hasta 1995, cuando alcanzó el $45 \%$ del total. Las exportaciones de productos de media y alta tecnología también vienen en aumento, y alcanzaron una participación del 33\% al final del período. Sin embargo, conviene destacar que, de ese $33 \%$, menos del $10 \%$ son productos de alta tecnología, lo que revela el predominio de bienes de media y baja tecnología en la producción nacional exportable.

En relación con las importaciones, el período 19811990 se caracteriza por la influencia de las crisis del 
GRÁFICO 1

Brasil: evolución de la participación sectorial de las exportaciones

(En porcentajes)

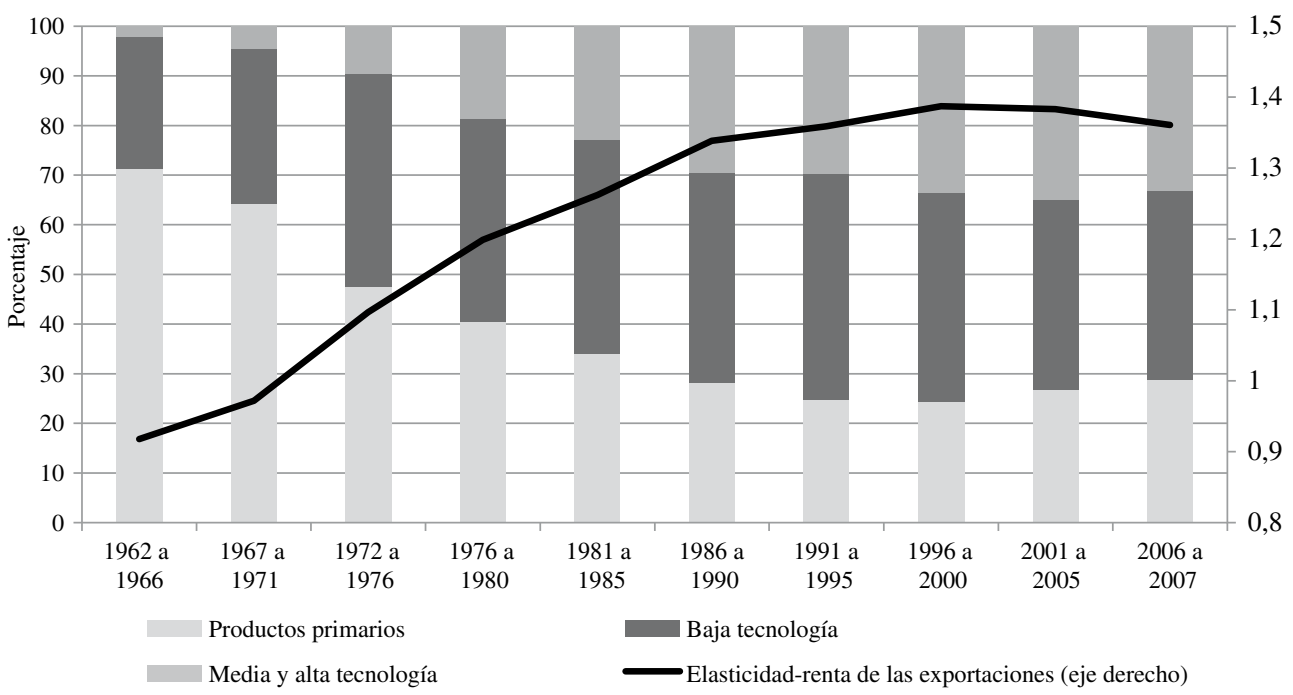

Fuente: elaboración propia a partir de la Base de datos estadísticos sobre el comercio de mercaderías (COMTRADE).

GRÁFICO 2

Brasil: evolución de la participación sectorial de las importaciones (En porcentajes)

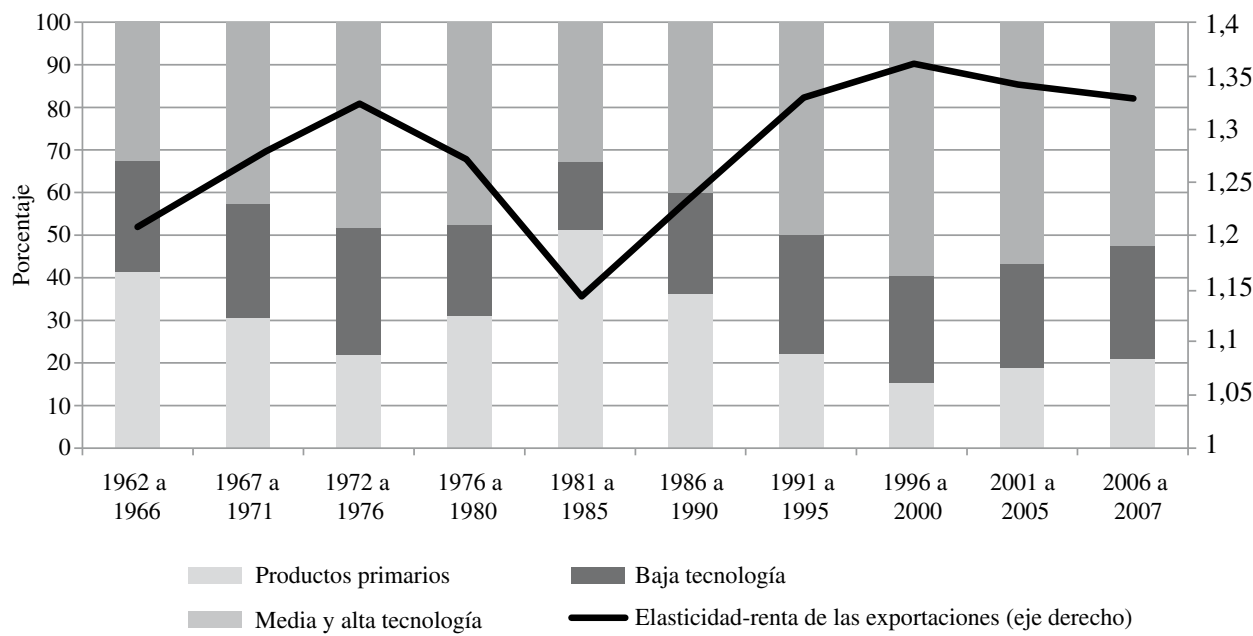

Fuente: elaboración propia a partir de la Base de datos estadísticos sobre el comercio de mercaderías (COMTRADE).

petróleo (sobre todo la segunda, en 1979), que impulsó el fuerte incremento del valor de las importaciones de productos primarios. Durante el resto del período, las importaciones de bienes de baja tecnología presentan cierta estabilidad y mantienen una participación en torno del $25 \%$. Por su parte, las importaciones de bienes de media y alta tecnología muestran un notable aumento, pasando de una participación del $34 \%$ al $52 \%$ al final del período. Cabe subrayar que, de ese porcentaje, cerca del 20\% representa bienes de alta tecnología. 
En síntesis, en los gráficos 1 y 2 se demuestra que el cambio estructural de la economía brasileña no se completó plenamente, lo que significa que aún existe un gran potencial para la ampliación de la producción de bienes de media y, especialmente, alta tecnología. Finalmente, es necesario resaltar que en los referidos gráficos, las líneas negras indican la manera en que los cambios de las elasticidades-renta acompañan a los cambios en la composición sectorial de la economía. Pese al cambio estructural ocurrido entre 1962 y 1985 , en el gráfico 1 es posible observar que, desde 1986, la pauta de exportaciones brasileñas se basaba sobre todo en bienes intensivos en recursos naturales y productos primarios, mientras que los productos de media y alta tecnología aumentaron en la pauta de las importaciones. Precisamente, el proceso de modernización y diversificación productiva que venía sucediendo en el Brasil a partir de los años cincuenta y que puede observarse en el modelo de especialización comercial, se estanca a partir de finales de los años ochenta, período en que la pauta de exportaciones de productos primarios e intensivos en recursos naturales se mantiene, aproximadamente, en más del $50 \%$ del total exportado. El fenómeno opuesto se registra en la pauta de importaciones, donde los productos de alta y media intensidad tecnológica representan más del $50 \%$ del volumen total importado entre 1989 y 2009 (Jayme Jr. y Resende, 2009).

\section{IV}

\section{Análisis empírico}

\section{Cálculo de las elasticidades hipotéticas}

En la literatura económica, la estimación empírica de las elasticidades reales ha venido realizándose mediante diversos modos alternativos. No obstante, a menudo es posible verificar la incompatibilidad de los datos en relación con algunas economías y períodos. De esa manera, en algunos trabajos se trató de sugerir sustitutos para estas elasticidades, también llamadas elasticidades hipotéticas. La definición más usual para dichas elasticidades es presentada por McCombie (1997), que define la "elasticidad renta hipotética" como aquella que iguala a las tasas de crecimiento observadas y teóricas: $\pi^{\prime} \equiv x / y^{6}$. Un segundo sustituto para las elasticidades puede derivarse de esta misma manera, siguiendo sin embargo las especificaciones del modelo presentado por Moreno-Brid (2003). Jayme Jr. (2003), a su vez, estima una "elasticidad implícita", $\pi$ ", que se obtiene a través del coeficiente de cointegración estimado a partir de la relación $\ln Y_{t}=\left(1 / \pi^{\prime \prime}\right) \ln X_{t}$.

Por lo tanto, las elasticidades hipotéticas pueden expresarse de la siguiente forma:

\footnotetext{
${ }^{6}$ De esta especificación se desprende que si $\pi$ ' y la estimación de $\pi$ no son estadísticamente diferentes, es imposible refutar la hipótesis de que el crecimiento del país resulta constreñido por la balanza de pagos (Santos, Lima y Carvalho, 2005). La estimación de $\pi$, a su vez, será ilustrada en la próxima subsección.
}

1) $\pi 1=x / y($ modelo original $)$

2) $\begin{aligned} \pi 2 \\ \text { (modelo Moreno-Brid) }\end{aligned}$

3) $\pi 3=1 / \beta$ obtenido por la cointegración de $\ln \mathrm{Y}_{\mathrm{t}}=\beta \ln \mathrm{X}_{\mathrm{t}}$

donde $x, y$ y $r$ se expresan en la tasa promedio de crecimiento del período analizado, y $\theta 1$ y $\theta 2$ se calculan para el período inicial.

Para verificar la adecuación de estas estimaciones al caso en estudio, se calcularon esas elasticidades para el Brasil entre 1962 y $2007^{7}$. Los datos en dólares para el PIB, las exportaciones y las importaciones se obtuvieron de la base de datos Ipeadata del Instituto de Pesquisa Económica Aplicada y de la Base de datos estadísticos sobre el comercio de mercaderías (COMTRADE). Los valores calculados para las elasticidades hipotéticas se encuentran resumidos en el cuadro 1.

Se observa que los resultados son similares, donde sobresale el hecho de que el modelo Moreno-Brid presenta valores levemente superiores a los del modelo original. Esa diferencia sería consecuencia de la no incorporación de los flujos de capitales en el modelo original, lo que

\footnotetext{
${ }^{7}$ Se adoptó tal período para que esos cálculos fueran compatibles con los datos utilizados en las pruebas del resto del trabajo.
} 
CUADRO 1

\section{Elasticidades hipotéticas}

\begin{tabular}{lcc}
\hline Tipo & $\begin{array}{c}\text { Datos de la } \\
\text { Ipeadata }\end{array}$ & $\begin{array}{c}\text { Datos de la } \\
\text { COMTRADE }\end{array}$ \\
\hline Modelo original & 1,112641455 & 1,029140941 \\
Modelo Moreno-Brid (2003) & 1,157374802 & 1,185313709 \\
Elasticidad implícita & 1,225173393 & 1,185973163 \\
\hline
\end{tabular}

Fuente: elaboración propia.

Nota: las especificaciones del modelo regresivo para el cálculo de la elasticidad implícita son las mismas que las de los modelos que se presentarán a continuación, y las estadísticas de las pruebas fueron sólidas.

COMTRADE: Base de datos estadísticos sobre el comercio de mercaderías. Ipeadata: Base de datos económicos y financieros, mantenida por el Instituto de Pesquisa Económica Aplicada (IPEA) del Brasil.

da como resultado la subestimación de la elasticidad. Esas estimaciones se utilizan como referencia inicial para la magnitud esperada de las elasticidades reales, obtenidas a través de procedimientos econométricos que se presentarán en la próxima subsección.

\section{Cálculo de las elasticidades reales totales y sectoriales}

En esta subsección se analiza la metodología utilizada en la estimación de las elasticidades de las exportaciones e importaciones brasileñas. Al considerar la hipótesis planteada en este estudio de que el principal determinante de las elasticidades es el grado tecnológico de la producción, se procuró descomponer las "elasticidades totales" de la economía entre los sectores de la producción nacional, según las categorías tecnológicas de su producto. Este abordaje sectorial — por categorías tecnológicas — de los bienes comercializados permite poner a prueba la hipótesis, ya que se espera que las elasticidades-renta, tanto de las importaciones como de las exportaciones, de los sectores de mayor intensidad tecnológica sean más elevadas que las de aquellos de baja tecnología y productos básicos.

En las pruebas realizadas se utilizan datos desagregados sectorialmente, según la Clasificación Uniforme para el Comercio Internacional (CUCI), de 2 y 3 dígitos, entre 1962 y 2007, respecto de las importaciones y exportaciones brasileñas disponibles en la base de datos COMTRADE. Los datos del PIB se obtuvieron de la Ipeadata (valores en dólares estadounidenses) y el cambio real se calculó a partir del tipo de cambio nominal proporcionado por la Ipeadata, dividido por la paridad del poder de compra (disponible en la base de datos Penn World Table) durante el período analizado ${ }^{8}$. Esa forma de cálculo se mostró más acorde con el análisis histórico del cambio real brasileño, puesto que entre 1962 y 1990 el cálculo usual $(E p f / p d)$ alcanza valores muy próximos a cero, lo que le hace perder poder explicativo. Las cuentas de la CUCI se agregaron de acuerdo con el cuadro 2.

A partir de esta clasificación, se estimaron modelos diferentes para cada una de las categorías de importaciones y exportaciones designadas de la siguiente manera: i) manufacturas de media y alta tecnología, de ahora en adelante $\mathrm{M} 1$ y X1 para importaciones y exportaciones, respectivamente; ii) manufacturas de baja tecnología o basadas en recursos naturales, $\mathrm{m} 2$ y x2, respectivamente; iii) productos básicos internacionales, $\mathrm{M} 3$ y X3, respectivamente, $\mathrm{y}$ iv) total de importaciones (M0) y exportaciones (X0). Las ecuaciones básicas a estimar son, por lo tanto, las siguientes funciones de demanda de importaciones $\mathrm{y}$ exportaciones originales de la ley de Thirlwall:

$$
\begin{aligned}
& \ln \mathrm{M}(\mathrm{i})=\beta_{0}+\beta_{1} \ln \mathrm{R}+\beta_{2} \ln \mathrm{Y} \operatorname{con} i \in(0,3) \\
& \ln \mathrm{X}(\mathrm{i})=\beta_{0}+\beta_{1} \ln \mathrm{R}+\beta_{2} \ln \mathrm{Z} \operatorname{con} i \in(0,3)
\end{aligned}
$$

donde $i \in(0,3)$ representa las diferentes categorías tecnológicas, $\mathrm{M}$ las importaciones, $\mathrm{X}$ las exportaciones, $\mathrm{R}$ el tipo de cambio real, $\mathrm{Y}$ la renta interna y $\mathrm{Z}$ la renta externa.

\section{- Metodología de estimación}

Se dice que un conjunto de series es cointegrado de orden $p-q-C I(p, q)-$ si i) todas las series son integradas de orden $\mathrm{p}-\mathrm{I}(\mathrm{p})-$, y ii) una combinación lineal entre ellas es integrada de orden $\mathrm{p}-\mathrm{q}$, siendo $q>0$. De esa forma, inicialmente se llevaron a cabo las pruebas para identificar la estacionalidad de las series en estudio. Aunque adoptada usualmente, la prueba de Dickey-Fuller aumentada (ADF) es muy sensible a la cantidad de desfases incluidos en el modelo. Además, la prueba toma como presupuesto la no autocorrelación y homocedasticidad de los residuos de su ecuación. Por lo tanto, ante la ausencia de normalidad de los residuos

\footnotetext{
${ }^{8}$ Se realizaron las mismas pruebas utilizando otros sustitutos para el tipo de cambio real, como el empleado por Hausman, Hwang y Rodrik (2005), r=1/p, y el tipo de cambio real calculado a partir del producto del tipo de cambio nominal por el cociente entre el índice de precios al por mayor norteamericano (IPA) y el IPC brasileño (Gouvêa y Lima, 2009). Los resultados encontrados fueron todos similares. La opción por la versión presentada responde a la mejor adecuación de la serie al análisis histórico del cambio brasileño, puesto que se trata de recurrentes procesos inflacionarios y modificaciones en los regímenes cambiarios durante el período en estudio.
} 
Brasil: agregación de datos de comercio reportados por la COMTRADE

\begin{tabular}{|c|c|c|c|c|c|c|c|c|c|}
\hline \multicolumn{2}{|c|}{$\begin{array}{l}\text { Productos } \\
\text { primarios }\end{array}$} & \multicolumn{3}{|c|}{$\begin{array}{l}\text { Manufacturas basadas en } \\
\text { recursos naturales }\end{array}$} & \multicolumn{2}{|c|}{$\begin{array}{l}\text { Manufacturas de } \\
\text { baja intensidad } \\
\text { tecnológica }\end{array}$} & \multicolumn{2}{|c|}{$\begin{array}{c}\text { Manufacturas } \\
\text { de intensidad } \\
\text { tecnológica media }\end{array}$} & \multirow{2}{*}{ 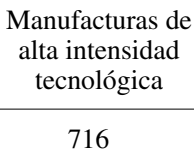 } \\
\hline 1 & 268 & 12 & 628 & 688 & 611 & 692 & 781 & 721 & \\
\hline 11 & 271 & 14 & 633 & 689 & 612 & 693 & 782 & 722 & 718 \\
\hline 22 & 273 & 23 & 634 & & 613 & 694 & 783 & 723 & 751 \\
\hline 25 & 274 & 24 & 635 & & 651 & 695 & 784 & 724 & 752 \\
\hline 34 & 277 & 35 & 641 & & 652 & 696 & 785 & 725 & 759 \\
\hline 36 & 278 & 37 & 281 & & 654 & 697 & 266 & 726 & 761 \\
\hline 41 & 291 & 46 & 282 & & 655 & 699 & 267 & 727 & 764 \\
\hline 42 & 292 & 47 & 286 & & 656 & 821 & 512 & 728 & 771 \\
\hline 43 & 322 & 48 & 287 & & 657 & 893 & 513 & 736 & 774 \\
\hline 44 & 333 & 56 & 288 & & 658 & 894 & 533 & 737 & 776 \\
\hline 45 & 341 & 58 & 289 & & 659 & 895 & 553 & 741 & 778 \\
\hline 54 & 681 & 61 & 323 & & 831 & 897 & 554 & 742 & 524 \\
\hline 57 & 682 & 62 & 334 & & 842 & 898 & 562 & 743 & 541 \\
\hline 71 & 683 & 73 & 335 & & 843 & 899 & 572 & 744 & 712 \\
\hline 72 & 684 & 98 & 411 & & 844 & & 582 & 745 & 792 \\
\hline 74 & 685 & 111 & 511 & & 845 & & 583 & 749 & 871 \\
\hline 75 & 686 & 112 & 514 & & 846 & & 584 & 762 & 874 \\
\hline 81 & 687 & 122 & 515 & & 847 & & 585 & 763 & 881 \\
\hline 91 & & 233 & 516 & & 848 & & 591 & 772 & \\
\hline 121 & & 247 & 522 & & 851 & & 598 & 773 & \\
\hline 211 & & 248 & 523 & & 642 & & 653 & 775 & \\
\hline 212 & & 251 & 531 & & 665 & & 671 & 793 & \\
\hline 222 & & 264 & 532 & & 666 & & 672 & 812 & \\
\hline 223 & & 265 & 551 & & 673 & & 678 & 872 & \\
\hline 232 & & 269 & 592 & & 674 & & 786 & 873 & \\
\hline 244 & & 423 & 661 & & 675 & & 791 & 884 & \\
\hline 245 & & 424 & 662 & & 676 & & 882 & 885 & \\
\hline 246 & & 431 & 663 & & 677 & & 711 & 951 & \\
\hline 261 & & 621 & 664 & & 679 & & 713 & & \\
\hline 263 & & 625 & 667 & & 691 & & 714 & & \\
\hline
\end{tabular}

Fuente: elaboración propia sobre la base de S. Lall, Competitiveness, Technology and Skills, Cheltenham, Edward Elgar. Publishing, 2001. Nota: clasificación de los productos según la Clasificación Uniforme para el Comercio Internacional (CUCI) a tres dígitos.

COMTRADE: Base de datos estadísticos sobre el comercio de mercaderías.

de la ecuación de la prueba ADF, la prueba de PhillipsPerron (PP), basada en un proceso estocástico MA(1), presenta mejores resultados.

En el anexo 1 de este trabajo se resumen las estadísticas de las pruebas ADF y PP para uno y tres desfases de las series en nivel y en primera diferencia. La elección de la cantidad de desfases se basó en el criterio de normalidad de los residuos de la ecuación ADF. Por consiguiente, los mejores resultados para un intervalo son aquellos presentados por la prueba $\mathrm{PP}$, mientras que para tres desfases la prueba ADF presenta mayor poder. Como se puede observar, para todas las variables en estudio se acepta la hipótesis nula de no estacionalidad en nivel. A la vez, se rechaza la misma hipótesis para las primeras diferencias, lo que confirma que las series en estudio son I(1), es decir, integradas de orden uno, permitiendo que probemos la existencia de relaciones a largo plazo entre ellas.
Por tratarse de un método de más fácil aplicación (en una única etapa), se optó por efectuar el llamado procedimiento de Johansen (Enders, 1995) para verificar la cointegración de las series y estimar su vector de largo plazo. Este procedimiento evita regresiones espurias y permite la estimación de parámetros consistentes para el modelo. La elección de la especificación de los modelos a probar se basó en la minimización de los criterios de información más utilizados por la literatura: criterio de información de Schwartz (SIC), criterio de información de Akaike (AIC), criterio de información de Hannan Quinn (HQC) y el error final de predicción (EFP). Tales criterios se estimaron utilizando un número máximo de rezagos en el sexto intervalo debido a la cantidad reducida de grados de libertad de los modelos, y sus resultados se encuentran resumidos en los anexos 2 y 3. Además, respecto de cada modelo se reportaron los resultados para las estadísticas de la traza (que señalan el número 
de vectores de cointegración entre las series) en los anexos 6 y 7, y pruebas de normalidad (autocorrelación y heteroscedasticidad) de los residuos para cada una de las especificaciones postuladas como relación de largo plazo en los anexos 4 y 5 .

Los resultados para los vectores de cointegración se presentan en la próxima subsección. Para todos los modelos se estimaron las siguientes especificaciones: i) sin constante, ii) con tendencia, y iii) con constante en el vector de cointegración. No obstante, solo se reportan los resultados para el modelo con constante en el vector de cointegración, que presentó resultados más sólidos en las pruebas.

Con el objeto de identificar las relaciones a corto plazo y la causalidad entre las variables, se optó por desarrollar un vector de corrección de errores (VEC). Debido a la estructura del VEC a estimar, a diferencia del modelo de autorregresión vectorial (VAR) del que deriva, la estimación por mínimos cuadrados ordinarios (MCO) no es adecuada, pues es necesario imponer restricciones cruzadas sobre el sistema de ecuaciones. Aunque sus resultados no se presenten, serán fundamentales para el análisis de las repercusiones de las innovaciones en el sistema.

Se emplearán dos instrumentos de análisis de las innovaciones: las funciones de impulso-respuesta y la descomposición de error final de predicción. El primer instrumento permite simular el comportamiento a lo largo del tiempo de las $n$ variables del modelo cuando ocurre una perturbación (shock) en los residuos de cada una de las variables bajo análisis. Esto es posible debido a la correlación parcial entre los residuos de cada una de las series pertenecientes al modelo, aunque se asuma que cualquier variación en estos residuos provendrá de perturbaciones exógenas. Dado el corto intervalo de convergencia de las series, los gráficos de la función impulso-respuesta abarcan un período de apenas 10 años. El segundo instrumento, la descomposición del error final de predicción del modelo, es complementario del primero en tanto permite analizar dinámicamente el comportamiento de las variables sujetas a perturbaciones, y muestra en cada período el peso de los residuos en el error final de predicción de los modelos. Dados el intervalo anual de los datos y su relativamente rápida convergencia, se ilustrarán resultados seleccionados para los primeros 20 períodos.

\section{Resultados}

\section{a) Elasticidades-renta de las importaciones \\ Inicialmente se admitió la siguiente relación a largo plazo: $m(i)=r+y$ (las letras minúsculas representan}

variables en logaritmo). Luego de que los criterios de información divergieron en cuanto a la especificación ideal para los modelos de cada una de las categorías de importación, se estimaron todos los modelos sugeridos por estos criterios. En el anexo 2 se muestra el número de desfases (denotados por " $p$ ") ideal para la VAR según cada criterio. De acuerdo con lo que se observa para el modelo más genérico, de las importaciones brasileñas como un todo (M0), el modelo ideal varió entre 1 y 5 desfases. De esa manera, se llevaron a cabo pruebas de normalidad, autocorrelación y heteroscedasticidad para los residuos de estas estimaciones (véase el anexo 4). La especificación final del VEC se escogió teniendo en cuenta todas las pruebas relatadas para cada una de las categorías de importaciones. A fin de garantizar la estandarización y permitir la comparación de las elasticidades de cada categoría de importaciones, se adoptó el modelo con 3 desfases $(p=3)$. Aunque el análisis de las pruebas anteriores pueda, en algunos casos, indicar el mejor ajuste de otras especificaciones, el vector de cointegración estimado no presentó una gran sensibilidad a las diferentes especificaciones, lo que justifica la opción por la estandarización de los vectores de cointegración relatados. Según se aprecia en el anexo 6, para todas las categorías de importación las estadísticas de la traza señalan la existencia de, por lo menos, una relación de cointegración entre las variables. Los vectores normalizados de cointegración se presentan en el cuadro 3.

Pese a que la interpretación de los coeficientes en vectores de cointegración es siempre arriesgada, las variables fueron significativas en todos los modelos y los coeficientes indican que los bienes importados de alta/media y baja tecnología (M1 y M2) poseen elasticidad-renta similares. Solo en el caso de los productos básicos (M3) se nota una diferencia significativa de umbral, lo que es compatible con el referencial teórico que indica una menor elasticidad-renta de la demanda de bienes primarios. Tales resultados pueden sugerir una relativa debilidad de la industria nacional, incluso de bienes de baja tecnología, ya que los aumentos de la renta promueven incrementos más que proporcionales de la demanda de estos bienes externos. Además, es notorio que las elasticidades estimadas son plenamente compatibles con las hipotéticas, calculadas anteriormente.

A objeto de garantizar la solidez de los parámetros, se estimaron nuevos vectores autorregresivos para cada categoría tecnológica, imponiendo sin embargo restricciones sobre el valor de las elasticidades-renta e igualándolas con las correspondientes a otras categorías. De esa forma, fue posible realizar pruebas de la razón de 
CUADRO 3

\section{Vector de cointegración}

Elasticidad-renta de MO

\begin{tabular}{llcll}
\hline Vector & $m$ & $y$ & $r$ & Constante \\
\hline Coeficiente & 1 & $-1,39057$ & 1,255712 & 12,09121 \\
Dp & & 0,104241 & 0,10314 & \\
Alpha & $-0,05863$ & 0,176316 & $-0,2299$ & \\
\hline
\end{tabular}

Elasticidad-renta de M1

\begin{tabular}{llccc}
\hline Coeficiente & 1 & $-1,45359$ & 2,394258 & 13,35274 \\
Dp & & 0,101666 & 0,097003 & \\
Alpha & $-0,06075$ & 0,074304 & $-0,12344$ & \\
\hline
\end{tabular}

Elasticidad-renta de M2

\begin{tabular}{llcll}
\hline Coeficiente & 1 & $-1,47117$ & 1,681609 & 15,40699 \\
Dp & & 0,097724 & 0,009242 & \\
Alpha & $-0,05752$ & $-0,2162$ & 0,093195 & \\
\hline
\end{tabular}

Elasticidad-renta de M3

\begin{tabular}{llcll}
\hline Coeficiente & 1 & $-0,84967$ & $-1,79363$ & 1,421589 \\
Dp & & 0,116154 & 0,012731 & \\
alpha & $-1,31621$ & $-0,18706$ & $-0,0016$ & \\
\hline
\end{tabular}

Fuente: elaboración propia.

Nota: 3 rezagos.

Dp: desviación estándar.

Alpha: coeficiente de velocidad de ajuste.

M0:total de importaciones.

M1:importaciones de manufacturas de media y alta tecnología.

M2: importaciones de manufacturas de baja tecnología o basadas en recursos naturales.

M3:importaciones de productos básicos internacionales.

verosimilitud ${ }^{9}$ para cada uno de los vectores estimados, a fin de verificar su "singularidad" estadística, es decir, garantizar la diferencia estadística de las elasticidades estimadas para cada categoría tecnológica. Los resultados de estas pruebas se presentan en el anexo 8. Sin embargo, cabe destacar que solo al comparar las elasticidades-renta de las importaciones de manufacturas de alta tecnología (M1) y de baja tecnología (M2) no se rechazó la hipótesis nula de igualdad estadística de los parámetros, según indica el $p$-valor de la prueba. En los demás casos, se rechaza al 5\% de significancia

\footnotetext{
${ }^{9}$ La prueba de la razón de verosimilitud se obtiene a partir de la comparación entre modelos con y sin las restricciones que son probadas. Por lo tanto, la hipótesis nula establece que cada uno de los parámetros en prueba sea igual al valor predefinido. La estadística de la prueba compara el valor obtenido con el de una distribución del chi-cuadrado con $(p-r) r l$ grados de libertad, donde $r$ es el número total de relaciones de cointegración verificadas, $p$ el número de líneas de la matriz de restricción sobre los betas (igual a 1) y $r l$ el número de columnas de esa misma matriz (igual al número de parámetros del modelo utilizado).
}

la igualdad de los parámetros, lo que confirma que las elasticidades-renta son diferentes para cada nivel de intensidad tecnológica.

Dado que el objetivo de este trabajo es analizar las elasticidades-renta, se revelarán los coeficientes encontrados para las elasticidades-precio (efectos del cambio real). No obstante, conviene resaltar la relación decreciente de estas con el nivel tecnológico de los bienes comercializados (el signo de los coeficientes es invertido). Un resultado que llama la atención es el hecho de que el signo de esta elasticidad sea contrario al esperado solo para el vector de los productos básicos. Aunque inusual, tal resultado es ampliamente consistente con los datos y con todos los modelos alternativos estimados: $\operatorname{VAR}(0)$, $\operatorname{VAR}(1), \operatorname{MCO}(1)$. Una hipótesis para este comportamiento del parámetro es que la devaluación cambiaria puede derivar en el incremento de las importaciones de productos básicos, en virtud de la necesidad de estos para la producción de bienes comercializables. Otra hipótesis posible es que la utilización de ingresos de importaciones presenta el sesgo del precio. La utilización de un índice de quantum importado puede alterar el resultado de este parámetro. La búsqueda de una explicación para ese comportamiento constituye una interesante agenda de investigación futura.

En el gráfico 3 se ilustran las funciones de impulsorespuesta para el modelo de importaciones agregado (M0) ${ }^{10}$. El análisis de estas innovaciones permite visualizar las relaciones a corto plazo entre las variables $\mathrm{y}$, por lo tanto, establecer también sus relaciones de causalidad, además de permitir, junto con la descomposición de la varianza del error, un análisis de los mecanismos dinámicos de propagación de los efectos de perturbaciones exógenas en las variables de cada modelo.

Del gráfico 3 se desprende que una perturbación exógena de un desvío estándar en las importaciones repercute poco en las demás variables del modelo, mostrando una relación positiva con la renta y negativa con el tipo de cambio real. Dicha perturbación es prácticamente consumida en los dos primeros períodos. Por otra parte, una perturbación en el tipo de cambio real (segunda columna) tiene un efecto desfasado en un período, pero de gran repercusión (negativa) en las importaciones y relativamente menor (positiva) en la renta. El encadenamiento de las relaciones entre las variables amortiza la propagación de los efectos de la

\footnotetext{
${ }^{10}$ Las líneas grises en los gráficos de impulso-respuesta representan el intervalo de confianza del $95 \%$ generado a partir de un "remuestreo" (bootstrap) con 100 repeticiones.
} 
Brasil: funciones de impulso-respuesta de las importaciones

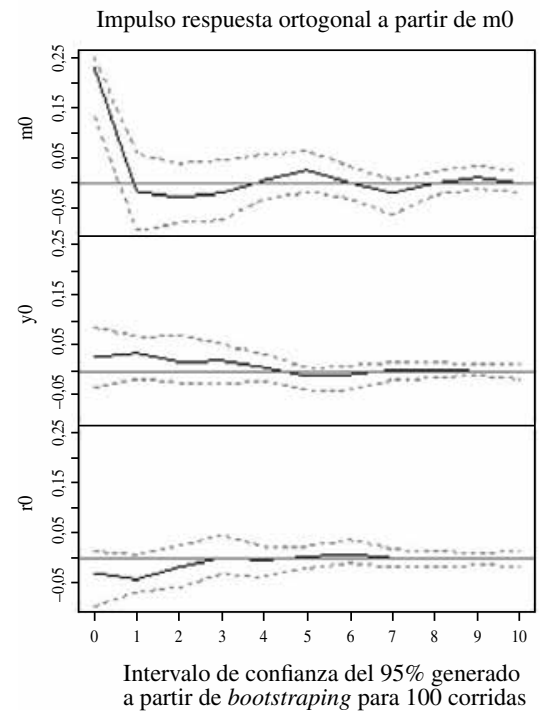

Fuente: elaboración propia.

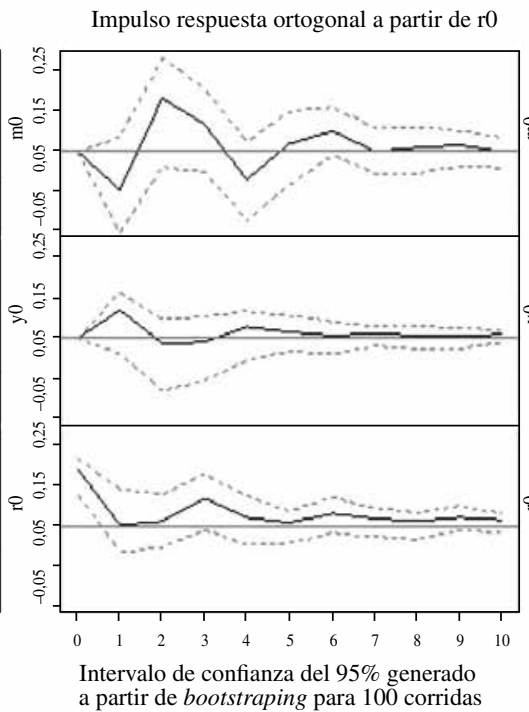

Impulso respuesta ortogonal a partir de y0

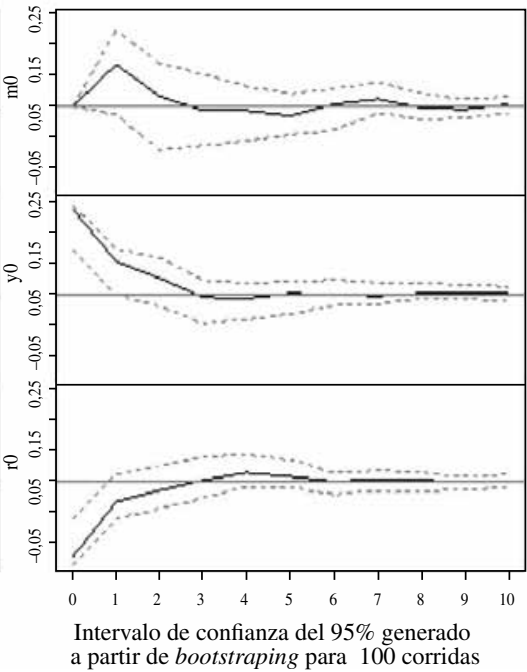

perturbación, que se extirpa completamente solo en el octavo período. A su vez, una perturbación exógena en la renta (tercera columna) no tiene un efecto contemporáneo en las importaciones, que responden (positivamente) solo en el período siguiente. Por su parte, el cambio real se aprecia de inmediato. Estos efectos se extinguen en el tercer período en una convergencia directa.

En el cuadro 4 se presentan los resultados para el análisis de la descomposición de los errores finales de predicción del modelo. Aunque la mayor parte del error final de predicción de $m$ se deba a sus propias innovaciones, estas pierden peso relativo tanto para $r$ (un desfase) como para $y$ (dos desfases) a lo largo del tiempo. En lo que respecta a $y$, mientras en el período vigente el $71 \%$ de su varianza derive de la variación de $r$ y solo el $25 \%$ de sus propias innovaciones, con 10 períodos tal proporción pasa al 58\% y al 31\%, respectivamente, quedando apenas una parte residual para $m$. Los errores finales de predicción de $r$ se derivan mayoritariamente de las innovaciones del propio cambio real. Sin embargo, a partir del período siguiente se nota un significativo aumento del peso relativo de $m$, que mantiene un $12 \%$ de participación en los errores del tipo de cambio a lo largo del tiempo, mientras $y$ pasa a ser importante continuamente.

\section{b) Elasticidad de las exportaciones}

Se propone ahora la siguiente relación a largo plazo: $x_{(i)}=r+z$. Tal como se procedió con las importaciones,
CUADRO 4

\section{Descomposición de la varianza-elasticidad de las importaciones}

\begin{tabular}{lcccc}
\hline \multirow{3}{*}{ Modelo } & & \multicolumn{3}{c}{ Innovaciones } \\
\cline { 3 - 5 } & & $m$ & $r$ & $y$ \\
\hline$m$ & 1 & 1,00 & 0,00 & 0,00 \\
& 2 & 0,88 & 0,12 & 0,00 \\
& 3 & 0,82 & 0,11 & 0,07 \\
& 4 & 0,81 & 0,12 & 0,07 \\
& 5 & 0,80 & 0,12 & 0,09 \\
& 10 & 0,79 & 0,12 & 0,09 \\
& 20 & 0,79 & 0,12 & 0,10 \\
\hline & 1 & 0,05 & 0,95 & 0,00 \\
& 2 & 0,12 & 0,87 & 0,01 \\
& 3 & 0,13 & 0,86 & 0,01 \\
& 4 & 0,12 & 0,83 & 0,05 \\
& 5 & 0,12 & 0,83 & 0,06 \\
& 10 & 0,12 & 0,81 & 0,07 \\
& 20 & 0,12 & 0,80 & 0,08 \\
\hline$y$ & 1 & 0,04 & 0,71 & 0,25 \\
& 2 & 0,08 & 0,60 & 0,32 \\
& 3 & 0,09 & 0,60 & 0,31 \\
& 4 & 0,10 & 0,59 & 0,31 \\
& 5 & 0,10 & 0,59 & 0,31 \\
& 10 & 0,11 & 0,58 & 0,31 \\
& 20 & 0,11 & 0,58 & 0,31 \\
\hline & & & &
\end{tabular}

Fuente: elaboración propia.

se efectuaron pruebas para la selección del modelo (véase el anexo 3): pruebas de normalidad, heteroscedasticidad y autocorrelación de los residuos de los modelos estimados 
CUADRO 5

\section{Vector de cointegración}

\section{Elasticidad-renta de XO}

\begin{tabular}{|c|c|c|c|c|}
\hline Vector & $x$ & $r$ & $z$ & Constante \\
\hline $\begin{array}{l}\text { Coeficiente } \\
\text { Dp } \\
\text { Alpha }\end{array}$ & $\begin{array}{c}1 \\
-0,02912\end{array}$ & $\begin{array}{r}-0,68115 \\
0,084565 \\
0,672952\end{array}$ & $\begin{array}{r}-1,14414 \\
0,035604 \\
0,047245\end{array}$ & 18,33868 \\
\hline \multicolumn{5}{|c|}{ Elasticidad-renta de X1 } \\
\hline $\begin{array}{l}\text { Coeficiente } \\
\text { Dp } \\
\text { Alpha }\end{array}$ & $\begin{array}{l}1 \\
0,047675\end{array}$ & $\begin{array}{r}-2,01321 \\
0,084257 \\
0,213471\end{array}$ & $\begin{array}{r}-1,9767 \\
0,036008 \\
0,020072\end{array}$ & 46,47997 \\
\hline
\end{tabular}

\section{Elasticidad-renta de X2}

\begin{tabular}{llccc}
\hline Coeficiente & 1 & $-0,96508$ & $-1,28721$ & 23,97728 \\
Dp & & 0,0869 & 0,00141 & \\
Alpha & 0,002171 & 0,297678 & 0,045457 & \\
\hline
\end{tabular}

Elasticidad-renta de X3

\begin{tabular}{llccc}
\hline Coeficiente & 1 & $-0,80188$ & $-0,74934$ & 7,619842 \\
Dp & & 0,082212 & 0,033953 & \\
Alpha & 0,062179 & 0,599276 & 0,020581 & \\
\hline
\end{tabular}

Fuente: elaboración propia.

Nota: 3 rezagos.

Dp: desviación estándar.

Alpha: coeficiente de velocidad de ajuste.

$\mathrm{X} 0$ : total de exportaciones.

$\mathrm{X} 1$ : exportaciones de manufacturas de media y alta tecnología.

$\mathrm{X} 2$ : exportaciones de manufacturas de baja tecnología o basadas en recursos naturales.

X3: exportaciones de productos básicos internacionales.

(véase el anexo 5) y pruebas de cointegración (véase el anexo 7). Sobre la base de las informaciones provenientes de estas pruebas, el modelo escogido para todos los casos quedaría entre las especificaciones con 2 y 3 desfases. En virtud de la similitud de los coeficientes estimados en ambos modelos, y para compatibilizar los análisis entre las elasticidades de las importaciones y exportaciones, se optó por la especificación con 3 desfases. Los vectores normalizados de cointegración para cada categoría de exportación se presentan en el cuadro 5.

Tomando en cuenta la excepción ya señalada en la interpretación de coeficientes en vectores de cointegración, se verifica que la elasticidad-renta de las exportaciones estimada es función creciente de la tecnología incorporada en los bienes exportados. Es más, se nota que la demanda de bienes brasileños de media y alta tecnología es significativamente elástica a las variaciones en la renta mundial, mientras que los productos básicos tienden a ser inelásticos respecto de la renta. Con las debidas salvedades ya referidas al examinar coeficientes en el análisis de cointegración, particularmente porque el vector de cointegración no establece una relación de causalidad entre las variables, tal resultado suscita importantes cuestiones, sobre todo debido a las grandes diferencias encontradas entre las elasticidades de cada categoría de bienes. En el anexo 9 se presentan los resultados de las pruebas de la razón de verosimilitud para las elasticidades-renta de las exportaciones. Las pruebas indican que las elasticidades-renta de cada sector son estadísticamente diferentes: se rechaza la hipótesis nula de que los coeficientes sean iguales (se subraya el caso de la elasticidad-renta de los bienes manufacturados de baja tecnología (X2) que solo es diferente de los demás al $10 \%$ de significancia).

Los resultados estimados sugieren que, al ser válida la restricción externa establecida por la ley de Thirlwall en cualquiera de sus versiones, una pauta de exportaciones más favorable a los bienes de mayor contenido tecnológico puede garantizar una tasa de crecimiento del producto superior a una pauta exportadora basada especialmente en productos básicos, como sucede de hecho en el caso brasileño.

En relación con las elasticidades-precio de la demanda de exportaciones, se advierte el mismo patrón encontrado en el caso de las importaciones: estas son directamente proporcionales al nivel de tecnología incorporada. Contrasta con ese resultado el diferente modelo de las elasticidades-renta de las importaciones y exportaciones. Las funciones de impulso-respuesta para la exportación agregada (X0) se ilustran en el gráfico 4.

Como se observa a partir del gráfico 4 , una perturbación exógena en las exportaciones provoca un efecto inmediato, pero poco significativo, tanto en la renta externa (positivo) como en el tipo de cambio real (negativo). Es necesario destacar el comportamiento contrario y desfasado entre $x$ y $r$. Las variaciones en el tipo de cambio real (segunda columna), a su vez, no tienen una incidencia significativa en las demás variables. Este resultado difiere enormemente del verificado para las importaciones, lo que indica un comportamiento asimétrico del tipo de cambio, que afecta más a las importaciones que a las exportaciones. Por otra parte, una perturbación exógena en la renta externa tiende gradualmente a aumentar las exportaciones en una proporción de 1:1 en el segundo período, cuando tal efecto comienza a desvanecerse. La respuesta del tipo de cambio es inversa a la de las exportaciones, se valoriza con el incremento de las exportaciones y se devalúa con su disminución.

En el cuadro 6 se ilustra la descomposición de errores finales de predicción para algunos períodos 


\section{Brasil: funciones de impulso-respuesta de las exportaciones}

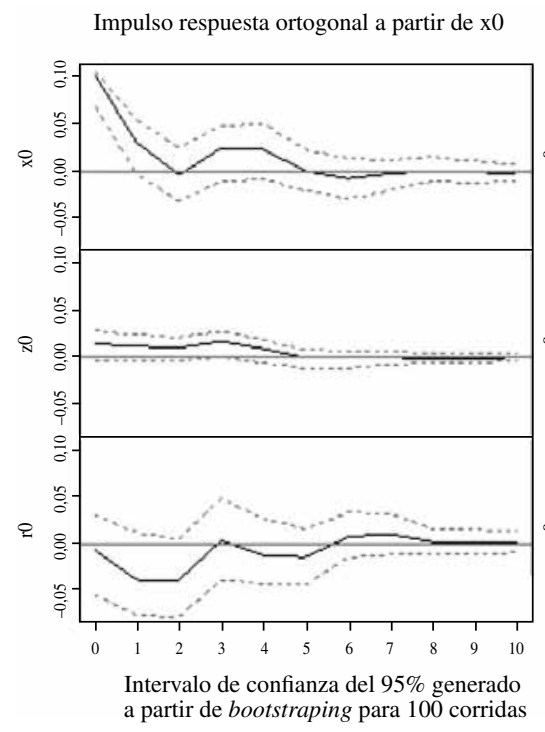

Impulso respuesta ortogonal a partir de r0

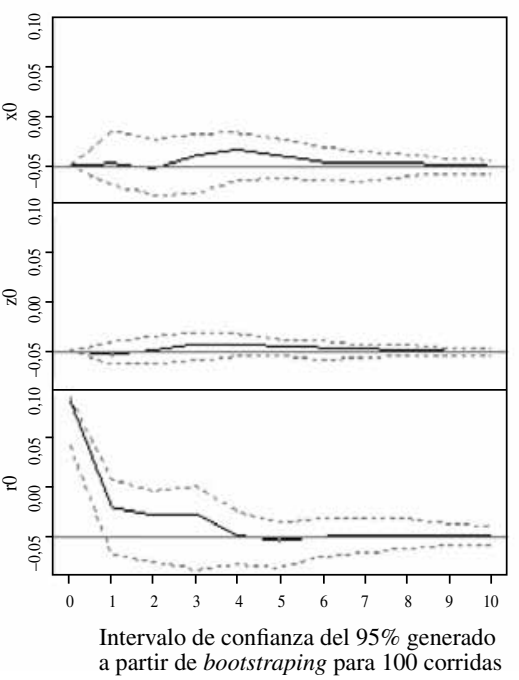

Impulso respuesta ortogonal a partir de z0

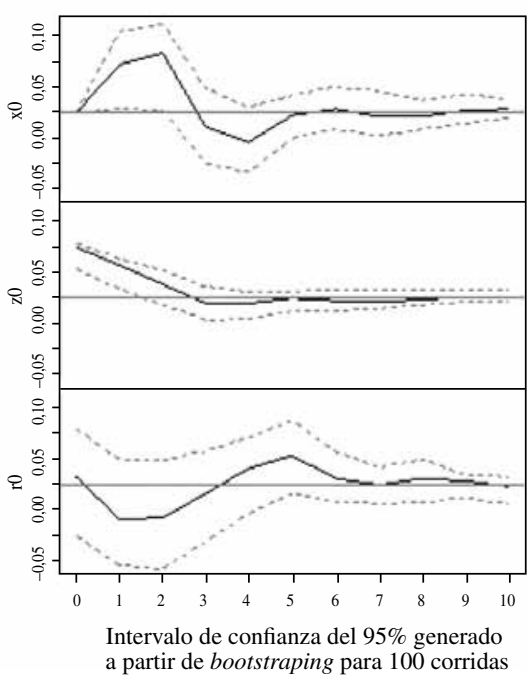

Fuente: elaboración propia.

CUADRO 6

\section{Descomposición de la varianza-elasticidad de las exportaciones}

\begin{tabular}{|c|c|c|c|c|}
\hline \multirow{2}{*}{ Modelo } & \multirow{2}{*}{ Período } & \multicolumn{3}{|c|}{ Innovaciones } \\
\hline & & $x$ & $r$ & $z$ \\
\hline \multirow[t]{7}{*}{$x$} & 1 & 1,00 & 0,00 & 0,00 \\
\hline & 2 & 0,89 & 0,00 & 0,11 \\
\hline & 3 & 0,76 & 0,00 & 0,24 \\
\hline & 4 & 0,76 & 0,01 & 0,23 \\
\hline & 5 & 0,73 & 0,02 & 0,25 \\
\hline & 10 & 0,73 & 0,02 & 0,25 \\
\hline & 20 & 0,73 & 0,02 & 0,25 \\
\hline \multirow[t]{7}{*}{$r$} & 1 & 0,00 & 1,00 & 0,00 \\
\hline & 2 & 0,07 & 0,90 & 0,03 \\
\hline & 3 & 0,12 & 0,82 & 0,06 \\
\hline & 4 & 0,11 & 0,82 & 0,06 \\
\hline & 5 & 0,12 & 0,81 & 0,07 \\
\hline & 10 & 0,13 & 0,79 & 0,09 \\
\hline & 20 & 0,13 & 0,78 & 0,09 \\
\hline \multirow[t]{7}{*}{$z$} & 1 & 0,12 & 0,00 & 0,88 \\
\hline & 2 & 0,14 & 0,00 & 0,86 \\
\hline & 3 & 0,16 & 0,00 & 0,83 \\
\hline & 4 & 0,24 & 0,02 & 0,74 \\
\hline & 5 & 0,25 & 0,04 & 0,71 \\
\hline & 10 & 0,24 & 0,05 & 0,71 \\
\hline & 20 & 0,24 & 0,05 & 0,71 \\
\hline
\end{tabular}

Fuente: elaboración propia. seleccionados del modelo. Entre los resultados, cabe destacar el gran peso de $z$ en la variación de $x$ ya a partir del tercer período, luego de una perturbación en $x$. Asimismo, según ya se destacó, es prácticamente nulo el efecto de la variación del cambio real en la variación de las exportaciones. A su vez, la variación del tipo de cambio, aunque sea predominantemente afectada por su propia innovación, con el tiempo responde a las pequeñas variaciones en $x$ que ella causó. En lo que respecta a los errores finales de predicción de $z$, resalta la gradual mayor gravitación de la varianza de las exportaciones en su explicación.

\section{c) Evolución de las elasticidades brasileñas}

De acuerdo con la ley de Thirlwall original $(y=\varepsilon z / \pi)$, cuanto mayor es la elasticidad-renta de la demanda de las exportaciones nacionales y menor la elasticidadrenta de las importaciones nacionales, mayor es la tasa de crecimiento compatible con el equilibrio a largo plazo en la balanza de pagos. Por medio de las pruebas aquí realizadas, se demuestra que cuanto mayor es el tenor tecnológico de la producción nacional, mayor es la elasticidad-renta de las exportaciones y, por otra parte, menor la elasticidad-renta de las importaciones. Tal cuadro se revierte en menores tasas de crecimiento compatibles con equilibrio en la balanza de pagos y menor alivio de la restricción externa al crecimiento. 
Un ejercicio simple, que ilustra bien este punto, consiste en simular la evolución de las tasas de crecimiento del producto brasileño compatibles con el equilibrio externo a partir de las elasticidades estimadas anteriormente. Utilizando las elasticidades estimadas, se verifican las tasas de crecimiento hipotéticas del producto suponiendo pautas de comercio exterior distintas para el Brasil: i) país especializado en exportaciones de alta tecnología e importaciones de bienes de baja tecnología y productos básicos; ii) país especializado en exportaciones de manufacturas de baja tecnología e importación de todos los tipos de bienes, y iii) país especializado en exportaciones de productos básicos e importaciones de manufacturas de toda especie. En el primer caso, la tasa promedio de crecimiento del producto año a año sería del orden del 6,75\%; del 3,67\% en el segundo y del $2,03 \%$ en el último. En contraste, el patrón brasileño efectivo presenta un crecimiento anual promedio del $3,26 \%$, lo que demuestra que el país estaría más cercano al segundo patrón propuesto.

Es destacable la semejanza del último caso con las tasas promedio de crecimiento efectivo del producto brasileño en la década de 1990, lo que sería previsible dada la forma de inserción del país en el comercio internacional. Llaman también la atención las diferencias de nivel de las tasas de crecimiento compatibles con el equilibrio en la balanza de pagos, de acuerdo con la estructura de comercio adoptada. Claramente, con la especialización en la exportación de bienes de alta tecnología se reduce la restricción externa al crecimiento del producto.

Sobre la base de las elasticidades estimadas para los diferentes niveles tecnológicos, según proponen
Gouvêa y Lima (2009), es posible analizar cómo las elasticidades del comercio brasileño se fueron modificando a lo largo del tiempo. En el gráfico 5 se señala de qué manera ese cambio se fue procesando año a año. Asimismo, se advierte que la elasticidad-renta de las importaciones permanece prácticamente en el mismo nivel de 1960 al final del período, pasando de 1,2\% en 1962 a 1,3\% en 2007. Al observar la tendencia de las importaciones, se percibe una propensión al aumento de la elasticidad-renta en el inicio del período, compatible con una mayor necesidad de importación de bienes de capital, que se revierte en el momento siguiente con el avance del modelo de industrialización por medio de la sustitución de importaciones. La fuerte caída de la década de 1980 obedece a los problemas de balanza de pagos enfrentados por el Brasil. A partir de 1990 la situación se revierte con una nueva elevación de la elasticidad-renta, que retorna al nivel inicial.

$\mathrm{Al}$ evaluar la evolución de la elasticidad-renta de las exportaciones el panorama se vuelve mucho más claro. Desde 1962 hasta el inicio de la década de 1990, se observa una constante elevación de la elasticidad, lo que indica un continuo cambio estructural orientado a sectores de mayor intensidad tecnológica, sobre todo de exportación de bienes primarios para las manufacturas de baja y media tecnología. A partir de 1990 se interrumpe el aumento de la elasticidad-renta de las exportaciones, que se mantiene estancada (pasa del 1,34\% en 1990 al $1,36 \%$ en 2007).

En el gráfico 6 esas elasticidades ponderadas se utilizan para el cálculo del crecimiento del PIB compatible con la estabilidad de la balanza de pagos (ley de

GRÁFICO 5

Brasil: evolución de las elasticidades ponderadas

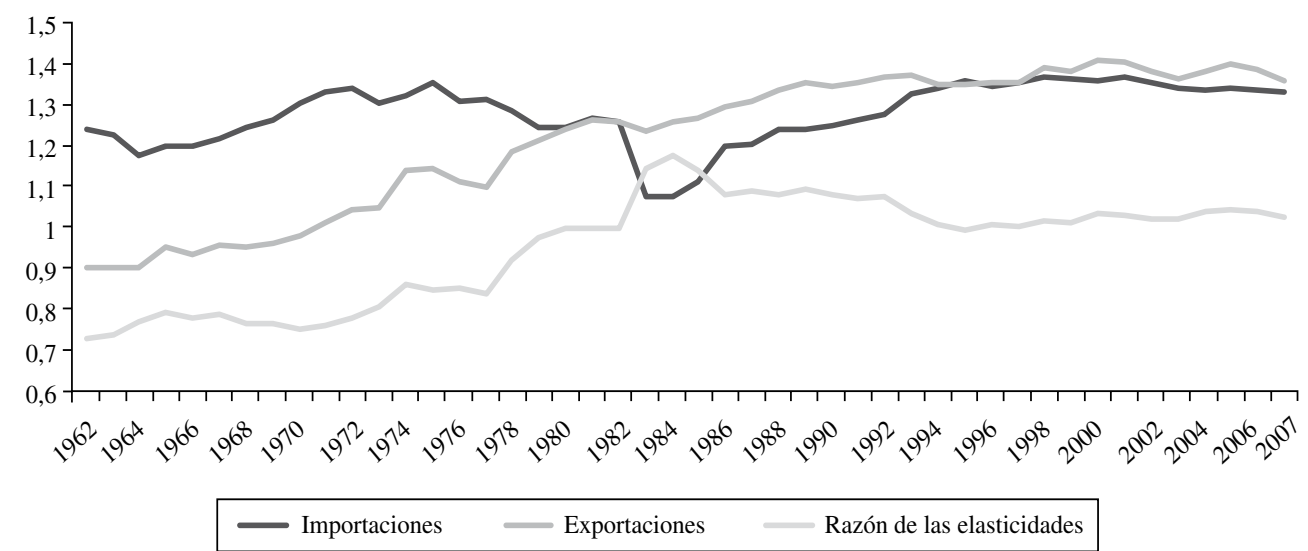

Fuente: elaboración propia. 
GRÁFICO 6

Brasil: crecimiento del PIB efectivo y estimado

(En porcentajes)

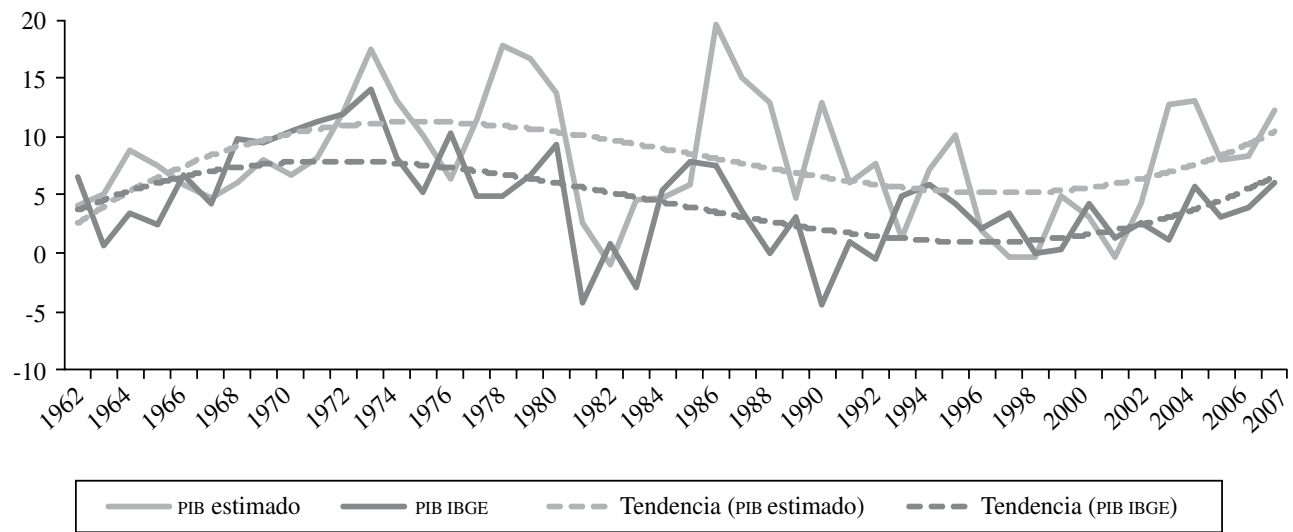

Fuente: elaboración propia.

Thirlwall). Como contrapunto al crecimiento del PIB estimado, se incorporó el crecimiento efectivo del PIB brasileño calculado por el IBGE.

En el gráfico 6 se constata que el crecimiento anual del PIB calculado según la ley de Thirlwall, sobre la base de las elasticidades ponderadas, es bastante similar al movimiento del PIB real. Sin embargo, se percibe que el crecimiento del PIB estimado se encuentra en un nivel más elevado que el crecimiento efectivamente verificado. Pero al analizar las líneas de tendencia se aprecia una gran similitud. Esta situación corrobora no

\section{$\mathrm{V}$}

\section{Conclusiones}

A lo largo de este artículo se procuró demostrar que el cambio estructural orientado a sectores productivos de mayor intensidad tecnológica reduce la restricción externa al crecimiento por medio de modificaciones en las elasticidades-renta de las importaciones y exportaciones.

Mediante la ley de Thirlwall se comprueba que, en última instancia, el nivel de crecimiento nacional se ve determinado por las elasticidades-renta de las demandas de importaciones y exportaciones. Por ende, mayores tasas de crecimiento guardan relación con una baja elasticidad-renta de las importaciones y con una elevada elasticidad-renta de las exportaciones. No obstante, usualmente tales variables se consideran exógenas en la literatura. Asimismo, Araujo y Lima (2007) y Gouvêa y solo la validez de la ley de Thirlwall, sino también la de las elasticidades sectoriales estimadas en el presente trabajo $^{11}$. También se demuestra que el cálculo de las elasticidades ponderadas es adecuado para analizar la evolución de las elasticidades-renta de las importaciones y exportaciones.

\footnotetext{
${ }^{11}$ Las pruebas de restricción realizadas por Gouvêa y Lima (2009) confirman la validez estadística de la llamada Ley de Thirlwall Multisectorial para la estimación del crecimiento efectivo del PIB.
}

Lima (2009) demuestran que los cambios en la estructura productiva producen cambios en las elasticidades, que son determinados directamente por el nivel de desarrollo tecnológico de la producción nacional. De acuerdo con Jayme Jr. y Resende (2009), los países desarrollados tienden a insertarse en el comercio internacional como proveedores de bienes manufacturados de media y alta tecnología e importadores de productos básicos y manufacturados de baja tecnología, situación inversa a la observada en países periféricos como el Brasil.

Para corroborar ese análisis, en el presente trabajo se desarrolló una serie de pruebas empíricas con que se procuró estimar las elasticidades-renta para categorías tecnológicas distintas de los bienes comercializables 
(tradables) brasileños, a partir de una adaptación de la clasificación propuesta por Lall (2001) con la reclasificación de los datos en tres grupos: i) productos básicos; ii) bienes de bajo tenor tecnológico y manufacturas basadas en recursos naturales, y iii) bienes de media y alta tecnología.

Los resultados de las pruebas corroboraron el marco teórico presentado, de modo que se confirmó la creciente relación entre el nivel tecnológico de las exportaciones y la elasticidad-renta, observándose lo mismo para las importaciones. De esa manera, se demuestra que se obtienen mayores niveles de crecimiento por medio de la inserción en el comercio mundial como exportador de bienes de media y alta tecnología (elevada elasticidad-renta) y como importador de bienes de baja tecnología (productos básicos, baja elasticidad-renta), que es exactamente el patrón verificado por Jayme Jr. y Resende (2009) para los países de la OCDE. Por lo tanto, es necesario que se produzca un cambio estructural dirigido a una mayor participación de la producción de sectores productores de bienes con elevado tenor tecnológico.
Al analizar separadamente la evolución de las elasticidades brasileñas, se observó el mantenimiento de un patrón importador similar, mientras que el cambio del perfil de las exportaciones verificadas hasta 1990 (que refleja el crecimiento de la elasticidad-renta de las exportaciones) se interrumpió a partir de entonces. Luego se observa el estancamiento del crecimiento de la elasticidad-renta de las exportaciones, es decir, una traba en el cambio estructural necesario para mantener un mayor crecimiento económico.

En las conclusiones se destaca la relevancia del desarrollo tecnológico como una forma de influir en las elasticidades y relajar así la restricción externa. Teniendo como referencia fundamental la importancia de mantener el crecimiento de la demanda para impulsar el crecimiento más acelerado del producto, se demostró aquí que la incorporación de tecnología en la producción (o el incremento de la participación de sectores de mayor tecnología en la producción nacional) es fundamental para mantener ese proceso y escapar de los problemas de la balanza de pagos.

ANEXO 1

Pruebas de raíz unitaria

\begin{tabular}{|c|c|c|c|c|c|c|c|c|}
\hline \multirow{2}{*}{$\begin{array}{c}\text { Variable } \\
\text { (en logaritmo natural) }\end{array}$} & \multirow{2}{*}{ Términos determinísticos } & \multicolumn{2}{|c|}{$\mathrm{ADF}$} & \multicolumn{2}{|c|}{ PP } & \multicolumn{3}{|c|}{ Valores críticos } \\
\hline & & $p=1$ & $p=3$ & $p=1$ & $p=3$ & $1 \%$ & $5 \%$ & $10 \%$ \\
\hline Logaritmo natural de exportaciones (X0) & $\begin{array}{l}\text { Constante } \\
\text { Constante, tendencia } \\
\text { Sin constante o tendencia }\end{array}$ & $\begin{array}{c}-1,0073 \\
-1,702 \\
3,1482 \\
\end{array}$ & $\begin{array}{r}-1,0856 \\
-1,9001 \\
2,2059 \\
\end{array}$ & $\begin{array}{c}-0,9815 \\
-1,4098 \\
- \\
\end{array}$ & $\begin{array}{l}-0,9713 \\
-1,4612 \\
- \\
\end{array}$ & $\begin{array}{l}-3,6 \\
-4,2 \\
-2,6 \\
\end{array}$ & $\begin{array}{l}-2,9 \\
-3,5 \\
-2 \\
\end{array}$ & $\begin{array}{l}-2,6 \\
-3,2 \\
-1,6 \\
\end{array}$ \\
\hline \multicolumn{2}{|c|}{$p$-valor $\Delta$ logaritmo natural exportaciones $(\Delta \mathrm{X} 0)$} & $-4,5247$ & $-1,9133$ & $-4,7894$ & $-4,6535$ & $-3,6$ & $-2,9$ & $-2,6$ \\
\hline $\begin{array}{l}\text { Logaritmo natural de exportaciones } \\
\text { media y alta tecnología (X1) }\end{array}$ & $\begin{array}{l}\text { Constante } \\
\text { Constante, tendencia } \\
\text { Sin constante o tendencia }\end{array}$ & $\begin{array}{r}-3,3928 \\
-1,7285 \\
3,4086 \\
\end{array}$ & $\begin{array}{r}-1,6306 \\
-1,3239 \\
1,4461 \\
\end{array}$ & $\begin{array}{c}-2,4835 \\
-1,0913 \\
- \\
\end{array}$ & $\begin{array}{l}-2,4958 \\
-1,0937 \\
- \\
\end{array}$ & $\begin{array}{l}-3,6 \\
-4,2 \\
-2,6 \\
\end{array}$ & $\begin{array}{l}-2,9 \\
-3,5 \\
-2 \\
\end{array}$ & $\begin{array}{l}-2,6 \\
-3,2 \\
-1,6 \\
\end{array}$ \\
\hline \multicolumn{2}{|c|}{$p$-valor $\Delta$ logaritmo natural exportaciones $(\Delta \mathrm{X} 1)$} & $-4,0831$ & $-2,1703$ & $-7,5037$ & $-6,4918$ & $-3,6$ & $-2,9$ & $-2,6$ \\
\hline $\begin{array}{l}\text { Logaritmo natural de exportaciones baja } \\
\text { tecnología y MBPP (X2) }\end{array}$ & $\begin{array}{l}\text { Constante } \\
\text { Constante, tendencia } \\
\text { Sin constante o tendencia }\end{array}$ & $\begin{array}{r}-1,5463 \\
-1,9023 \\
2,4001 \\
\end{array}$ & $\begin{array}{r}-1,4435 \\
-1,5597 \\
2,1264 \\
\end{array}$ & $\begin{array}{l}-1,7223 \\
-1,4776 \\
- \\
\end{array}$ & $\begin{array}{l}-1,7207 \\
-1,4891 \\
- \\
\end{array}$ & $\begin{array}{l}-3,6 \\
-4,2 \\
-2,6 \\
\end{array}$ & $\begin{array}{l}-2,9 \\
-3,5 \\
-2 \\
\end{array}$ & $\begin{array}{l}-2,6 \\
-3,2 \\
-1,6 \\
\end{array}$ \\
\hline \multicolumn{2}{|c|}{$p$-valor $\Delta$ logaritmo natural exportaciones $(\Delta \mathrm{X} 2)$} & $-4,552$ & $-2,508$ & $-4,1562$ & $-3,9967$ & $-3,6$ & $-2,9$ & $-2,6$ \\
\hline $\begin{array}{l}\text { Logaritmo natural de exportaciones de } \\
\text { productos básicos (X3) }\end{array}$ & $\begin{array}{l}\text { Constante } \\
\text { Constante, tendencia } \\
\text { Sin constante o tendencia }\end{array}$ & $\begin{array}{r}-0,1897 \\
-1,5201 \\
3,4335 \\
\end{array}$ & $\begin{array}{r}-0,388 \\
-1,8119 \\
2,3967 \\
\end{array}$ & $\begin{array}{l}-0,1508 \\
-1,507 \\
- \\
\end{array}$ & $\begin{array}{l}-0,1512 \\
-1,5691 \\
- \\
\end{array}$ & $\begin{array}{l}-3,6 \\
-4,2 \\
-2,6 \\
\end{array}$ & $\begin{array}{l}-2,9 \\
-3,5 \\
-2 \\
\end{array}$ & $\begin{array}{l}-2,6 \\
-3,2 \\
-1,6 \\
\end{array}$ \\
\hline \multicolumn{2}{|c|}{$p$-valor $\Delta$ logaritmo natural exportaciones $(\Delta \mathrm{X} 3)$} & $-4,7922$ & $-1,6117$ & $-6,2379$ & $-6,1631$ & $-3,6$ & $-2,9$ & $-2,6$ \\
\hline $\begin{array}{l}\text { Logaritmo natural de importaciones } \\
\text { (M0) }\end{array}$ & $\begin{array}{l}\text { Constante } \\
\text { Constante, tendencia } \\
\text { Sin constante o tendencia }\end{array}$ & $\begin{array}{r}-0,8408 \\
-2,3734 \\
2,8086 \\
\end{array}$ & $\begin{array}{r}-1,6391 \\
-2,2357 \\
3,0893 \\
\end{array}$ & $\begin{array}{c}-0,7095 \\
-2,3846 \\
- \\
\end{array}$ & $\begin{array}{l}-0,68 \\
-2,3635 \\
- \\
\end{array}$ & $\begin{array}{l}-3,6 \\
-4,2 \\
-2,6 \\
\end{array}$ & $\begin{array}{l}-2,9 \\
-3,5 \\
-2 \\
\end{array}$ & $\begin{array}{l}-2,6 \\
-3,2 \\
-1,6 \\
\end{array}$ \\
\hline \multicolumn{2}{|c|}{$p$-valor $\Delta$ logaritmo natural importaciones $(\Delta \mathrm{M})$} & $-5,4268$ & $-3,4345$ & $-6,5959$ & $-6,5873$ & $-3,6$ & $-2,9$ & $-2,6$ \\
\hline $\begin{array}{l}\text { Logaritmo natural de importaciones } \\
\text { media y alta tecnología (M1) }\end{array}$ & $\begin{array}{l}\text { Constante } \\
\text { Constante, tendencia } \\
\text { Sin constante o tendencia }\end{array}$ & $\begin{array}{r}-1,0652 \\
-2,2985 \\
2,8889 \\
\end{array}$ & $\begin{array}{r}-1,9014 \\
-3,1387 \\
2,6019 \\
\end{array}$ & $\begin{array}{c}-0,5777 \\
-1,9597 \\
- \\
\end{array}$ & $\begin{array}{l}-0,5922 \\
-2,1476 \\
- \\
\end{array}$ & $\begin{array}{l}-3,6 \\
-4,2 \\
-2,6 \\
\end{array}$ & $\begin{array}{l}-2,9 \\
-3,5 \\
-2 \\
\end{array}$ & $\begin{array}{l}-2,6 \\
-3,2 \\
-1,6 \\
\end{array}$ \\
\hline
\end{tabular}


(continuación anexo 1)

\begin{tabular}{|c|c|c|c|c|c|c|c|c|}
\hline \multirow{2}{*}{$\begin{array}{c}\text { Variable } \\
\text { (en logaritmo natural) }\end{array}$} & \multirow{2}{*}{ Términos determinísticos } & \multicolumn{2}{|c|}{$\mathrm{ADF}$} & \multicolumn{2}{|c|}{$\mathrm{PP}$} & \multicolumn{3}{|c|}{ Valores críticos } \\
\hline & & $p=1$ & $p=3$ & $p=1$ & $p=3$ & $1 \%$ & $5 \%$ & $10 \%$ \\
\hline \multicolumn{2}{|c|}{$p$-valor $\Delta$ logaritmo natural importaciones $(\Delta \mathrm{M} 1)$} & $-4,5227$ & $-2,7473$ & $-5,3069$ & $-5,2477$ & $-3,6$ & $-2,9$ & $-2,6$ \\
\hline $\begin{array}{l}\text { Logaritmo natural de importaciones baja } \\
\text { tecnología y MBPP (M2) }\end{array}$ & $\begin{array}{l}\text { Constante } \\
\text { Constante, tendencia } \\
\text { Sin constante o tendencia }\end{array}$ & $\begin{array}{r}-0,6095 \\
-2,6197 \\
2,3578 \\
\end{array}$ & $\begin{array}{r}-1,448 \\
-3,0147 \\
2,4985 \\
\end{array}$ & $\begin{array}{l}-0,6711 \\
-2,4366 \\
- \\
\end{array}$ & $\begin{array}{l}-0,6522 \\
-2,4935 \\
- \\
\end{array}$ & $\begin{array}{l}-3,6 \\
-4,2 \\
-2,6 \\
\end{array}$ & $\begin{array}{l}-2,9 \\
-3,5 \\
-2\end{array}$ & $\begin{array}{l}-2,6 \\
-3,2 \\
-1,6\end{array}$ \\
\hline \multicolumn{2}{|c|}{$p$-valor $\Delta$ logaritmo natural importaciones $(\Delta \mathrm{M} 2)$} & $-5,0339$ & $-3,2973$ & $-5,9736$ & $-5,8637$ & $-3,6$ & $-2,9$ & $-2,6$ \\
\hline $\begin{array}{l}\text { Logaritmo natural de importaciones } \\
\text { productos básicos (M3) }\end{array}$ & $\begin{array}{l}\text { Constante } \\
\text { Constante, tendencia } \\
\text { Sin constante o tendencia }\end{array}$ & $\begin{array}{r}-0,9294 \\
-2,1366 \\
1,9646 \\
\end{array}$ & $\begin{array}{c}-1,2343 \\
-1,4751 \\
2,641 \\
\end{array}$ & $\begin{array}{l}-0,9105 \\
-2,2766 \\
- \\
\end{array}$ & $\begin{array}{l}-0,8048 \\
-2,1473 \\
- \\
\end{array}$ & $\begin{array}{l}-3,6 \\
-4,2 \\
-2,6 \\
\end{array}$ & $\begin{array}{l}-2,9 \\
-3,5 \\
-2 \\
\end{array}$ & $\begin{array}{l}-2,6 \\
-3,2 \\
-1,6\end{array}$ \\
\hline \multicolumn{2}{|c|}{$p$-valor $\Delta$ logaritmo natural importaciones $(\Delta \mathrm{M} 3)$} & $-5,8363$ & $-3,7605$ & $-7,0592$ & $-7,2128$ & $-3,6$ & $-2,9$ & $-2,6$ \\
\hline Logaritmo natural de renta $(y)$ & $\begin{array}{l}\text { Constante } \\
\text { Constante, tendencia } \\
\text { Sin constante o tendencia }\end{array}$ & $\begin{array}{l}-1,2455 \\
-2,3293 \\
-2,5966 \\
\end{array}$ & $\begin{array}{r}-1,6134 \\
-2,5615 \\
2,1484 \\
\end{array}$ & $\begin{array}{l}-1,1976 \\
-1,6692 \\
- \\
\end{array}$ & $\begin{array}{l}-1,1781 \\
-1,8223 \\
- \\
\end{array}$ & $\begin{array}{l}-3,6 \\
-4,2 \\
-2,6 \\
\end{array}$ & $\begin{array}{l}-2,9 \\
-3,5 \\
-2 \\
\end{array}$ & $\begin{array}{l}-2,6 \\
-3,2 \\
-1,6\end{array}$ \\
\hline \multicolumn{2}{|l|}{$p$-valor $\Delta$ logaritmo natural renta $(\Delta y)$} & $-3,4504$ & $-3,3783$ & $-4,2903$ & $-4,2895$ & $-3,6$ & $-2,9$ & $-2,6$ \\
\hline Logaritmo natural de cambio real $(r)$ & $\begin{array}{l}\text { Constante } \\
\text { Constante, tendencia } \\
\text { Sin constante o tendencia }\end{array}$ & $\begin{array}{l}-1,8862 \\
-2,1321 \\
-1,073 \\
\end{array}$ & $\begin{array}{l}-2,2197 \\
-2,528 \\
-1,1633\end{array}$ & $\begin{array}{l}-1,7124 \\
-1,5413 \\
- \\
\end{array}$ & $\begin{array}{l}-1,8856 \\
-1,813 \\
- \\
\end{array}$ & $\begin{array}{l}-3,6 \\
-4,2 \\
-2,6 \\
\end{array}$ & $\begin{array}{l}-2,9 \\
-3,5 \\
-2 \\
\end{array}$ & $\begin{array}{l}-2,6 \\
-3,2 \\
-1,6 \\
\end{array}$ \\
\hline \multicolumn{2}{|c|}{$p$-valor $\Delta$ logaritmo natural cambio real $(\Delta r)$} & $-3,5253$ & $-3,348$ & $-4,7811$ & $-4,901$ & $-3,6$ & $-2,9$ & $-2,6$ \\
\hline Logaritmo natural de renta mundial $(z)$ & $\begin{array}{l}\text { Constante } \\
\text { Constante, tendencia } \\
\text { Sin constante o tendencia }\end{array}$ & $\begin{array}{r}-1,2938 \\
-1,7455 \\
2,8775 \\
\end{array}$ & $\begin{array}{c}-1,5925 \\
-1,396 \\
2,5457 \\
\end{array}$ & $\begin{array}{l}-1,9428 \\
-0,9759 \\
- \\
\end{array}$ & $\begin{array}{l}-1,7883 \\
-1,0934 \\
- \\
\end{array}$ & $\begin{array}{l}-3,6 \\
-4,2 \\
-2,6 \\
\end{array}$ & $\begin{array}{l}-2,9 \\
-3,5 \\
-2 \\
\end{array}$ & $\begin{array}{l}-2,6 \\
-3,2 \\
-1,6 \\
\end{array}$ \\
\hline \multicolumn{2}{|c|}{$p$-valor $\Delta$ logaritmo natural renta mundial $(\Delta z)$} & $-3,4193$ & $-2,0366$ & $-3,3719$ & $-3,4036$ & $-3,6$ & $-2,9$ & $-2,6$ \\
\hline
\end{tabular}

Fuente: elaboración propia.

Nota 1: Los valores críticos de las pruebas ADF son aquellos reportados en D. Dickey y W.A. Fuller "Likelihood ratio statistics for autoregressive time series with a unit root", Econometrica, vol. 49, N 4, Nueva York, Econometric Society, junio de 1981; y J.D. Hamilton, Time Series Analysis, Princeton, Princeton University Press, 1994.

Nota 2: H0 (hipótesis nula de las pruebas: existencia de raíz unitaria).

Nota 3: los valores reportados se refieren a la estadística tal.

PP: prueba de Phillips-Perron.

MBPP: Manufacturas basadas en productos primarios.

$\Delta:$ diferencia o variación.

$\mathrm{X} 0$ : exportaciones totales.

$\mathrm{X} 1$ : exportaciones de manufacturas de media y alta tecnología.

$\mathrm{X} 2$ : exportaciones de manufacturas de baja tecnología o basadas en recursos naturales.

$\mathrm{X} 3$ : exportaciones de productos básicos internacionales.

M0: importaciones totales.

M1:importaciones de manufacturas de media y alta tecnología.

M2:importaciones de manufacturas de baja tecnología o basadas en recursos naturales.

M3:importaciones de productos básicos internacionales. 
ANEXO 2

\section{Selección del orden de la VAR}

\begin{tabular}{lcccc}
\hline Elasticidad-renta de XO & \multicolumn{3}{l}{} \\
\hline Rezago & AIC $(p)$ & $\operatorname{HQC}(p)$ & $\operatorname{SIC}(p)$ & $\operatorname{FPE}(p)$ \\
\hline Tendencia & 2 & 2 & 2 & 2 \\
Intercepto & 4 & 2 & 2 & 2 \\
Tendencia e intercepto & 2 & 2 & 2 & 2 \\
Ninguno & 2 & 2 & 2 & 2 \\
\hline Elección & \multicolumn{5}{c}{3 rezagos } \\
\hline
\end{tabular}

Elasticidad-renta de X1

\begin{tabular}{lllll}
\hline Tendencia & 2 & 2 & 2 & 2 \\
Intercepto & 2 & 2 & 2 & 2 \\
Tendencia e intercepto & 3 & 2 & 2 & 2 \\
Ninguno & 2 & 2 & 2 & 2 \\
\hline Elección & \multicolumn{5}{c}{3 rezagos } \\
\hline
\end{tabular}

\section{Elasticidad-renta de X2}

\begin{tabular}{lllll}
\hline Tendencia & 2 & 2 & 2 & 2 \\
Intercepto & 2 & 2 & 2 & 2 \\
Tendencia e intercepto & 2 & 2 & 2 & 2 \\
Ninguno & 2 & 2 & 2 & 2 \\
\hline Elección & \multicolumn{5}{c}{3 rezagos } \\
\hline
\end{tabular}

\begin{tabular}{lllll}
\hline Elección & \multicolumn{3}{l}{3 rezagos } \\
\hline Elasticidad-renta de X3 & 6 & 6 & 1 & 6 \\
\hline Tendencia & 6 & 6 & 1 & 6 \\
Intercepto & 6 & 5 & 1 & 5 \\
Tendencia e intercepto & 6 & 2 & 1 & 6 \\
Ninguno & & & 3 rezagos
\end{tabular}

Elección

3 rezagos

Fuente: elaboración propia.

Nota: número máximo de rezagos $=6$.

VAR: modelo de autorregresión vectorial. AIC: criterio de información de Akaike. HQC: criterio de información de Hannan Quinn. SIC: criterio de información de Schwarz. EFP: error final de predicción.

$\mathrm{X} 0$ : exportaciones totales.

$\mathrm{X} 1$ : exportaciones de manufacturas de media y alta tecnología.

$\mathrm{X} 2$ : exportaciones de manufacturas de baja tecnología o basadas en recursos naturales.

X3: exportaciones de productos básicos internacionales.
ANEXO 3

\section{Selección del orden de la VAR}

\begin{tabular}{|c|c|c|c|c|}
\hline \multicolumn{5}{|l|}{ Elasticidad-renta de MO } \\
\hline Rezago & $\operatorname{AIC}(p)$ & $\operatorname{HQC}(p)$ & $\operatorname{SIC}(p)$ & $\operatorname{FPE}(p)$ \\
\hline Tendencia & 5 & 1 & 1 & 2 \\
\hline Intercepto & 5 & 2 & 1 & 2 \\
\hline Tendencia e intercepto & 5 & 2 & 1 & 2 \\
\hline Ninguno & 5 & 1 & 1 & 2 \\
\hline Elección & \multicolumn{4}{|c|}{3 rezagos } \\
\hline \multicolumn{5}{|l|}{ Elasticidad-renta de M1 } \\
\hline Tendencia & 5 & 2 & 1 & 5 \\
\hline Intercepto & 4 & 2 & 1 & 4 \\
\hline Tendencia e intercepto & 6 & 4 & 1 & 4 \\
\hline Ninguno & 4 & 2 & 1 & 4 \\
\hline Elección & \multicolumn{4}{|c|}{3 rezagos } \\
\hline \multicolumn{5}{|l|}{ Elasticidad-renta de M2 } \\
\hline Tendencia & 5 & 1 & 1 & 5 \\
\hline Intercepto & 5 & 5 & 1 & 5 \\
\hline Tendencia e intercepto & 5 & 5 & 1 & 5 \\
\hline Ninguno & 5 & 1 & 1 & 5 \\
\hline Elección & \multicolumn{4}{|c|}{3 rezagos } \\
\hline \multicolumn{5}{|l|}{ Elasticidad-renta de M3 } \\
\hline Tendencia & 5 & 1 & 1 & 2 \\
\hline Intercepto & 5 & 2 & 1 & 2 \\
\hline Tendencia e intercepto & 5 & 2 & 1 & 2 \\
\hline Ninguno & 2 & 2 & 1 & 2 \\
\hline Elección & \multicolumn{4}{|c|}{3 rezagos } \\
\hline
\end{tabular}

Fuente: elaboración propia.

Nota: número máximo de rezagos $=6$.

VAR: modelo de autorregresión vectorial.

AIC: criterio de información de Akaike.

HQC: criterio de información de Hannan Quinn.

SIC: criterio de información de Schwarz.

EPF: error final de predicción.

M0: importaciones totales.

M1: importaciones de manufacturas de media y alta tecnología.

M2: importaciones de manufacturas de baja tecnología o basadas en recursos naturales.

M3: importaciones de productos básicos internacionales. 
ANEXO 4

Diagnóstico de los residuos

\begin{tabular}{|c|c|c|c|c|c|c|}
\hline \multicolumn{7}{|c|}{ Elasticidad-renta de MO } \\
\hline Modelo & JB & $p$-valor & Q & $p$-valor & $\mathrm{ARCH}$ & $p$-valor \\
\hline$p=3$ & 24,2956 & 0,0004607 & 101,818 & 0,7221 & 82,0855 & 0,1951 \\
\hline$p=2$ & 19,1771 & $2,51 x-10^{4}$ & 99,842 & 0,9096 & 95,4263 & 0,03381 \\
\hline$p=1$ & 38,7853 & $7,89 x-10^{7}$ & 107,595 & 0,915 & 100,0163 & 0,01617 \\
\hline \multicolumn{7}{|c|}{ Elasticidad-renta de $M 1$} \\
\hline$p=3$ & 14,1097 & 0,02843 & 91,0622 & 0,9166 & 62,7236 & 0,7742 \\
\hline$p=2$ & 18,8462 & $4,43 \times 10^{3}$ & 103,18 & 0,8638 & 69,9512 & 0,5464 \\
\hline$p=1$ & 25,1554 & $3,20 \times 10^{4}$ & 116,412 & 0,779 & 73,3348 & 0,4341 \\
\hline \multicolumn{7}{|c|}{ Elasticidad-renta de M2 } \\
\hline$p=3$ & 6,0231 & 0,4206 & 103,307 & 0,6857 & 89,8549 & 0,0757 \\
\hline$p=2$ & 12,7499 & 0,04718 & 101,144 & 0,8932 & 95,6307 & 0,03276 \\
\hline$p=1$ & 21,5636 & 0,001452 & 110,03 & 0,8854 & 106,9891 & 0,004691 \\
\hline \multicolumn{7}{|c|}{ Elasticidad-renta de M3 } \\
\hline$p=3$ & 24,9692 & 0,000346 & 111,948 & 0,4569 & 86,4326 & 0,1179 \\
\hline$p=2$ & 29,839 & $4,22 \times 10^{5}$ & 109,363 & 0,7469 & 120,7874 & 0,000281 \\
\hline$p=1$ & 58,9255 & $7,44 \times 10^{11}$ & 104,191 & 0,9468 & 118,1968 & 0,000493 \\
\hline
\end{tabular}

Fuente: elaboración propia.

Nota: resultados referidos al mejor modelo con intercepto en el vector de cointegración.

Jarque-Bera (JB): prueba de Jarque-Bera de normalidad de los residuos (H0: residuos normales).

Portmanteau (Q): prueba para autocorrelación en los residuos (H0: no-autocorrelación).

ARCH: prueba de heterocedasticidad condicional regresiva en los residuos (H0: homocedasticidad).

M0:importaciones totales.

M1:importaciones de manufacturas de media y alta tecnología.

M2:importaciones de manufacturas de baja tecnología o basadas en recursos naturales.

M3:importaciones de productos básicos internacionales.
ANEXO 5

\section{Diagnóstico de los residuos}

\begin{tabular}{|c|c|c|c|c|c|c|}
\hline \multicolumn{7}{|c|}{ Elasticidad-renta de XO } \\
\hline Modelo & JB & $p$-valor & Q & $p$-valor & $\mathrm{ARCH}$ & $p$-valor \\
\hline$p=3$ & 6,2999 & 0,3904 & 105,107 & 0,6397 & 76,529 & 0,3354 \\
\hline$p=2$ & 10,8343 & 0,09364 & 98,5533 & 0,924 & 59,0846 & 0,8626 \\
\hline$p=1$ & 9,5393 & $1,45 \times 10^{1}$ & 104,955 & 0,9406 & 82,9702 & 0,1771 \\
\hline \multicolumn{7}{|c|}{ Elasticidad-renta de XI } \\
\hline$p=3$ & 1,7535 & 0,941 & 90,9865 & 0,9175 & 59,843 & 0,8461 \\
\hline$p=2$ & 3,8717 & 0,694 & 96,6295 & 0,9424 & 51,5521 & 0,9672 \\
\hline$p=1$ & 9,4118 & $1,52 \times 10^{1}$ & 83,0726 & 0,9994 & 78,4628 & 0,2815 \\
\hline \multicolumn{7}{|c|}{ Elasticidad-renta de X2 } \\
\hline$p=3$ & 5,3618 & 0,4983 & 109,938 & 0,5107 & 72,3643 & 0,4658 \\
\hline$p=2$ & 11,212 & 0,08204 & 90,9576 & 0,9777 & 68,3158 & 0,6012 \\
\hline$p=1$ & 8,7636 & 0,1873 & 90,6287 & 0,9958 & 86,4695 & 0,1174 \\
\hline \multicolumn{7}{|c|}{ Elasticidad-renta de X3 } \\
\hline$p=6$ & 3,0402 & 0,8038 & 99,9439 & 0,113 & 65,6792 & 0,6869 \\
\hline$p=3$ & 2,4965 & 0,8689 & 101,418 & 0,7316 & 67,873 & 0,616 \\
\hline$p=2$ & 7,6678 & 0,2635 & 99,2726 & 0,9162 & 82,3719 & 0,1892 \\
\hline
\end{tabular}

Fuente: elaboración propia.

Nota: resultados referidos al mejor modelo con intercepto en el vector de cointegración.

Jarque-Bera (Јв): prueba de Jarque-Bera de normalidad de los residuos (H0: residuos normales).

Portmanteau (Q): prueba para autocorrelación en los residuos ( $\mathrm{H} 0$ : no-autocorrelación).

ARCH: prueba de heterocedasticidad condicional regresiva en los residuos (H0: homocedasticidad).

$\mathrm{X} 0$ : exportaciones totales.

$\mathrm{X} 1$ : exportaciones de manufacturas de media y alta tecnología.

$\mathrm{X} 2$ : exportaciones de manufacturas de baja tecnología o basadas en recursos naturales.

X3: exportaciones de productos básicos internacionales. 
ANEXO 6

\section{Prueba de cointegración}

\begin{tabular}{|c|c|c|c|c|c|c|}
\hline \multicolumn{7}{|c|}{ Elasticidad-renta de $M O$} \\
\hline \multirow{2}{*}{ H0 } & \multicolumn{3}{|c|}{ Estadísticas de la prueba } & \multicolumn{3}{|c|}{ Valores críticos } \\
\hline & $p=1$ & $p=2$ & $p=3$ & $90 \%$ & $95 \%$ & $99 \%$ \\
\hline$r=0$ & 39,07 & 34,12 & 36,75 & 32 & 34,91 & 41,07 \\
\hline$r=1$ & 16,86 & 17,67 & 17,73 & 17,85 & 19,96 & 24,6 \\
\hline$r=2$ & 6,52 & 4,14 & 6,12 & 7,52 & 9,24 & 12,97 \\
\hline \multicolumn{7}{|c|}{ Elasticidad-renta de $M 1$} \\
\hline$r=0$ & 35,81 & 36,47 & 46,42 & 32 & 34,91 & 41,07 \\
\hline$r=1$ & 15,37 & 17,59 & 20,99 & 17,85 & 19,96 & 24,6 \\
\hline$r=2$ & 4,79 & 2,35 & 6,07 & 7,52 & 9,24 & 12,97 \\
\hline \multicolumn{7}{|c|}{ Elasticidad-renta de $M 2$} \\
\hline$r=0$ & 39,83 & 40,01 & 42,37 & 32 & 34,91 & 41,07 \\
\hline$r=1$ & 17,59 & 21,14 & 20,41 & 17,85 & 19,96 & 24,6 \\
\hline$r=2$ & 7,72 & 4,45 & 7,51 & 7,52 & 9,24 & 12,97 \\
\hline \multicolumn{7}{|c|}{ Elasticidad-renta de M3 } \\
\hline$r=0$ & 44,47 & 39,56 & 35,67 & 32 & 34,91 & 41,07 \\
\hline$r=1$ & 18,55 & 19,34 & 15,69 & 17,85 & 19,96 & 24,6 \\
\hline$r=2$ & 5,05 & 8,44 & 6,87 & 7,52 & 9,24 & 12,97 \\
\hline
\end{tabular}

Fuente: elaboración propia.

Nota 1: resultados referidos al mejor modelo con intercepto en el vector de cointegración.

Nota 2: resultados referidos a las estadísticas de la traza.

Nota 3: los valores críticos de las estadísticas de la traza se refieren a aquellos encontrados en S. Johansen, Likelihood-Based Inference in Cointegrated Vector Autoregressive Models, Nueva York, Oxford University Press, 1995.

H0: la hipótesis nula es que existen $r$ vectores de cointegración. M0: importaciones totales.

M1: importaciones de manufacturas de media y alta tecnología.

M2: importaciones de manufacturas de baja tecnología o basadas en recursos naturales.

M3: importaciones de productos básicos internacionales.
ANEXO 7

\section{Prueba de cointegración}

\begin{tabular}{|c|c|c|c|c|c|c|}
\hline \multicolumn{7}{|c|}{ Elasticidad-renta de XO } \\
\hline \multirow{2}{*}{ H0 } & \multicolumn{3}{|c|}{ Estadísticas de la prueba } & \multicolumn{3}{|c|}{ Valores críticos } \\
\hline & $p=1$ & $p=2$ & $p=3$ & $90 \%$ & $95 \%$ & $99 \%$ \\
\hline$r=0$ & 36,44 & 40,27 & 48,21 & 32 & 34,91 & 41,07 \\
\hline$r=1$ & 9,7 & 11,87 & 16,46 & 17,85 & 19,96 & 24,6 \\
\hline$r=2$ & 2,98 & 2,17 & 4,79 & 7,52 & 9,24 & 12,97 \\
\hline \multicolumn{7}{|c|}{ Elasticidad-renta de X1 } \\
\hline$r=0$ & 45,69 & 61,08 & 51,54 & 32 & 34,91 & 41,07 \\
\hline$r=1$ & 20,41 & 29,3 & 20,8 & 17,85 & 19,96 & 24,6 \\
\hline$r=2$ & 6,53 & 7,21 & 4,47 & 7,52 & 9,24 & 12,97 \\
\hline \multicolumn{7}{|c|}{ Elasticidad-renta de X2 } \\
\hline$r=0$ & 40,84 & 41,87 & 40,64 & 32 & 34,91 & 41,07 \\
\hline$r=1$ & 12,19 & 13,65 & 15,28 & 17,85 & 19,96 & 24,6 \\
\hline$r=2$ & 5,28 & 5,33 & 5,31 & 7,52 & 9,24 & 12,97 \\
\hline \multicolumn{7}{|c|}{ Elasticidad-renta de X3 } \\
\hline$r=0$ & 34,02 & 49,86 & 80,43 & 32 & 34,91 & 41,07 \\
\hline$r=1$ & 9,59 & 14,75 & 36,36 & 17,85 & 19,96 & 24,6 \\
\hline$r=2$ & 1,55 & 2,33 & 17,59 & 7,52 & 9,24 & 12,97 \\
\hline
\end{tabular}

Fuente: elaboración propia.

Nota 2: resultados referidos a la estadística de la traza.

Nota 3: los valores críticos de la estadística de la traza se refieren a aquellos encontrados en S. Johansen, Likelihood-Based Inference in Cointegrated Vector Autoregressive Models, Nueva York, Oxford University Press, 1995.

Nota 1: resultados referidos al modelo con intercepto en el vector de cointegración.

H0: hipótesis nula.

$\mathrm{X} 0$ : exportaciones totales.

$\mathrm{X} 1$ : exportaciones de manufacturas de media y alta tecnología.

$\mathrm{X} 2$ : exportaciones de manufacturas de baja tecnología o basadas en recursos naturales.

X3: exportaciones de productos básicos internacionales.

ANEXO 8

Prueba de la razón de verosimilitud

\begin{tabular}{|c|c|c|c|c|c|c|c|c|}
\hline \multirow{2}{*}{ Sector } & \multicolumn{2}{|c|}{ M0 } & \multicolumn{2}{|c|}{ M1 } & \multicolumn{2}{|c|}{ M2 } & \multicolumn{2}{|c|}{ M3 } \\
\hline & Prueba & $p$-valor & Prueba & $p$-valor & Prueba & $p$-valor & Prueba & $p$-valor \\
\hline M0 & - & - & 7,7 & 0,02 & 7,77 & 0,02 & 7,92 & 0,02 \\
\hline M1 & 10,98 & 0 & - & - & 3,36 & 0,19 & 11,14 & 0 \\
\hline M2 & 8,52 & 0,01 & 6,35 & 0,04 & - & - & 8,55 & 0,01 \\
\hline M3 & 6,63 & 0,04 & 6,63 & 0,04 & 6,63 & 0,04 & - & - \\
\hline
\end{tabular}

Fuente: elaboración propia.

Nota: la hipótesis nula de la prueba establece que los coeficientes bajo restricción (elasticidad-renta de las importaciones) son iguales en los modelos representados en cada línea y columna.

M0: importaciones totales

M1: importaciones de manufacturas de media y alta tecnología.

M2: importaciones de manufacturas de baja tecnología o basadas en recursos naturales.

M3: importaciones de productos básicos internacionales. 
ANEXO 9

Prueba de la razón de verosimilitud

\begin{tabular}{|c|c|c|c|c|c|c|c|c|}
\hline \multirow{2}{*}{ Sector } & \multicolumn{2}{|c|}{ X0 } & \multicolumn{2}{|c|}{$\mathrm{X} 1$} & \multicolumn{2}{|c|}{$\mathrm{X} 2$} & \multicolumn{2}{|c|}{ X3 } \\
\hline & Prueba & $p$-valor & Prueba & $p$-valor & Prueba & $p$-valor & Prueba & $p$-valor \\
\hline X0 & - & - & 5,72 & 0,06 & 5,73 & 0,06 & 5,71 & 0,06 \\
\hline $\mathrm{X} 1$ & 8,09 & 0,02 & - & - & 8,08 & 0,02 & 8,1 & 0,02 \\
\hline $\mathrm{X} 2$ & 5 & 0,08 & 5,07 & 0,08 & - & - & 5,05 & 0,08 \\
\hline X3 & 6,28 & 0,04 & 6,28 & 0,04 & 6,28 & 0,04 & - & - \\
\hline
\end{tabular}

Fuente: elaboración propia.

Nota: la hipótesis nula de la prueba establece que los coeficientes bajo restricción (elasticidad-renta de las exportaciones) son iguales en los modelos representados en cada línea y columna.

X0: exportaciones totales.

$\mathrm{X} 1$ : exportaciones de manufacturas de media y alta tecnología.

$\mathrm{X} 2$ : exportaciones de manufacturas de baja tecnología o basadas en recursos naturales.

$\mathrm{X} 3$ : exportaciones de productos básicos internacionales.

\section{Bibliografia}

Abramovitz, M. (1986), "Catching up, forging ahead, and falling behind", The Journal of Economic History, vol. 46, № 2, Nueva York, Cambridge University Press.

Albuquerque, E.M. (1999), "National systems of innovation and non-OECD countries: notes about a rudimentary and tentative 'typology'”, Brazilian Journal of Political Economy, vol. 19, $\mathrm{N}^{\circ}$ 4, São Paulo.

Araujo, R.A. y G.T. Lima (2007), "A structural economic dynamics approach to balance-of-payments-constrained growth", Cambridge Journal of Economics, vol. 31, № 5, Oxford, Oxford University Press.

Atesoglu, H.S. (1997), "Balance of payments constrained growth model and its implications for the United States", Journal of Post Keynesian Economics, vol. 19, № 3, Armonk, M.E. Sharpe.

Barbosa-Filho, N.H. (2001), "Balance-of-payments constraint: from balanced trade to sustainable debt", Banca Nazionale del Lavoro Quarterly Review, № 219, Roma, Banca Nazionale del Lavoro.

Bértola, L., H. Higachi y G. Porcile (2002), "Balance-of-payments constrained growth in Brazil: a test of Thirlwall's law, 19801973”, Journal of Post Keynesian Economics, vol. 25, № 1, Armonk, M.E. Sharpe.

Carvalho, V.R. y G.T. Lima (2008), "A restrição externa e a perda de dinamismo da economia brasileira: investigando relações entre estrutura produtiva e crescimento econômico", Anais do XXXV Encontro Nacional de Economia, Niterói, Asociación Nacional de Centros de Posgrado en Economía (ANPEC).

Dickey, D. y W.A. Fuller (1981), "Likelihood ratio statistics for autoregressive time series with a unit root", Econometrica, vol. 49, $\mathrm{N}^{\mathrm{o}}$ 4, Nueva York, Econometric Society, junio.

Enders, W. (1995), Applied Econometric Time Series, Nueva York, John Wiley \& Sons.

Fajnzylber, R.F. (2000), "Da caixa-preta ao conjunto vazio", Cinqüenta anos de pensamento na CEPAL, R. Bielschowsky (org.), Río de Janeiro, Record.

(1983), La industrialización trunca de América Latina, México, D.F., Nueva Imagen.

Ferreira, A. (2001), "A lei de crescimento de Thirlwall", disertación, Campinas, Instituto de Economía/Universidad Estadual de Campinas.

Freeman, C. (2004), “The 'National System of Innovation' in historical perspective", Revista brasileira de inovação, vol. $3, \mathrm{~N}^{\circ} 1$, Fondo para el Financiamiento de Estudios y Proyectos (FINEP).
Gouvêa, R.R. y G.T. Lima (2009), "Structural change, balance-ofpayments constraint and economic growth: evidence from the multi-sectoral Thirlwall's Law", Anais do XXXVII Encontro Nacional de Economia da ANPEC, Niterói, Asociación Nacional de Centros de Posgrado en Economía (ANPEC).

Hamilton, J.D. (1994), Time Series Analysis, Princeton, Princeton University Press.

Harrod, R. (1933), International Economics, Cambridge, Cambridge University Press.

Hausman, R., J. Hwang y D. Rodrik (2005), "What you export matters", NBER Working Papers, $\mathrm{N}^{\circ} 11905$, Cambridge, Massachusetts, National Bureau of Economic Research.

Holland, M., F. Vieira y O. Canuto (2004), "Economic growth and the balance-of-payments constraint in Latin America", Investigación económica, vol. 63, México, D.F., Universidad Nacional Autónoma de México.

Jayme Jr., F.G. (2003) "Balance-of-payments constrained economic growth in Brazil", Revista de economia política, vol. 23, São Paulo.

Jayme Jr., F.G. y M.F.C. Resende (2009), "Crescimento econômico e restrição externa: teoria e a experiência brasileira", Crescimento econômico: setor externo e inflação, R. Michel y L. Carvalho (orgs.), Río de Janeiro, Instituto de Investigación Económica Aplicada (IPEA).

Johansen, S. (1995), Likelihood-Based Inference in Cointegrated Vector Autoregressive Models, Nueva York, Oxford University Press.

Kaldor, N. (1966), Causes of the Slow Rate of Economic Growth of the United Kingdom, Londres, Cambridge University Press.

Lall, S. (2001), Competitiveness, Technology and Skills, Cheltenham, Edward Elgar Publishing.

López, J. y A. Cruz (2000), "Thirlwall's law and beyond: the Latin American experience", Journal of Post Keynesian Economics, vol. 22, No 3, Armonk, M.E. Sharpe.

Lundvall, B. (ed.) (1992), National Systems of Innovation: Towards a Theory of Innovation and Interactive Learning, Londres, Pinter.

McCombie, J.S.L. (1997), "On the empirics of balance-of-payments constrained growth", Journal of Post Keynesian Economics, vol. 19, No 3 , Armonk, M.E. Sharpe.

McCombie, J.S.L. y A.P. Thirlwall (1999), "Growth in an international context: a post Keynesian view", Foundations of International Economics: Post Keynesian Perspectives, J. Deprez y J.T. Harvey (orgs.), Londres, Routledge. 
(1997), "Economic growth and balance-of-payments constraint revisited", Markets, Unemployment and Economic Policy, P. Arestis, G. Palma y M. Sawyer (eds.), vol. 2, Londres, Routledge. (1994), Economic Growth and the Balance-of-Payments Constraint, Nueva York, St. Martin's Press.

Moreno-Brid, J. (2003), "Capital flows, interest payments and the balance-of-payments constrained growth model: a theoretical and empirical analysis", Metroeconomica, vol. 54, № 2-3, Wiley Blackwell.

Nelson, R.R. (ed.) (1993), National Innovation Systems: A Comparative Analysis, Oxford, Oxford University Press.

(1964), "Aggregate production functions and medium-range growth projections", American Economic Review, vol. 54, № 5 , Nashville, Tennessee, American Economic Association.

Pérez, C. y L. Soete (1988), "Catching up in technology: entry barriers and windows of opportunity", Technical Change and Economic Theory, Dosi y otros (eds.), Londres, Macmillan.

Porcile, G. y G.T. Lima (2006), "Tipo de cambio, empleo y crecimiento en un modelo con restricción externa”, Investigación económica, vol. 65, N 257, México, D.F., Universidad Nacional Autónoma de México.

Prebisch, R. (2000a), "O desenvolvimento da economia da América Latina e alguns de seus problemas principais", Cinqüenta anos de pensamento na CEPAL, R. Bielschowsky (org.), Río de Janeiro, Record. (2000b), "Problemas teóricos e práticos do crescimento econômico", Cinqüenta anos de pensamento na CEPAL, R. Bielschowsky (org.), Río de Janeiro, Record.

Resende, M.F.C. y D.R. Torres (2008), "National Innovation System, trade elasticities and economic growth", Anais do XXXVI Encontro Nacional de Economia, Niterói, Asociación Nacional de Centros de Posgrado en Economía (ANPEC).

Santos, A.T., G.T. Lima y V.R.S. Carvalho (2005), “A restrição externa como fator limitante do crescimento econômico brasileiro: um teste empírico", Anais do XXXIII Encontro Nacional de Economia, Natal [en línea] http://www.anpec.org.br.

Thirlwall, A.P. (1979), "The balance of payments constraint as an explanation of international growth rates differences", Banca Nazionale del Lavoro Quarterly Review, No 128, Roma, Banca Nazionale del Lavoro.

Thirlwall, A.P. y M. Hussain (1982), "The balance of payments constraint, capital flows and growth rates differences between developing countries", Oxford Economic Papers, № 10, Oxford, Oxford University Press.

Vieira, F.A. y M. Holland (2006), "Crescimento econômico secular no Brasil, modelo de Thirlwall e termos de troca", Anais do XXXIV Encontro Nacional de Economia da ANPEC, Niterói, Asociación Nacional de Centros de Posgrado en Economía (ANPEC), diciembre. 